\title{
Failure Mechanisms of Lithium Metal Anode and Their Advanced Characterization Technologies
}

\author{
Xinyang Yue ${ }^{1, \dagger}$, Cui Ma ${ }^{1, \dagger}$, Jian Bao ${ }^{1}$, Siyu Yang ${ }^{2}$, Dong Chen ${ }^{1}$, Xiaojing Wu ${ }^{1}$, Yongning Zhou ${ }^{1, *}$ \\ ${ }^{1}$ Department of Materials Science, Fudan University, Shanghai 200433, China. \\ ${ }^{2}$ Department of Chemistry, Fudan University, Shanghai 200433, China.
}

\begin{abstract}
Although traditional graphite anodes ensure the cycling stability and safety of lithium-ion batteries, the inherent drawbacks, particularly low theoretical specific capacity $\left(372 \mathrm{mAh} \cdot \mathrm{g}^{-1}\right)$ and Li-free character, of such anodes limit their applications in high energy density battery systems, especially in lithium-sulfur and lithium-air batteries. Lithium metal has been considered as one of the best next-generation anode materials due to its extremely high theoretical specific capacity $\left(3860 \mathrm{mAh} \cdot \mathrm{g}^{-1}\right)$ and low redox potential $(-3.04 \mathrm{~V}$ vs. the standard hydrogen electrode). The first generation of commercial rechargeable lithium metal batteries were developed by Moli Energy in the late 1980s and were not widely used due to several problems,

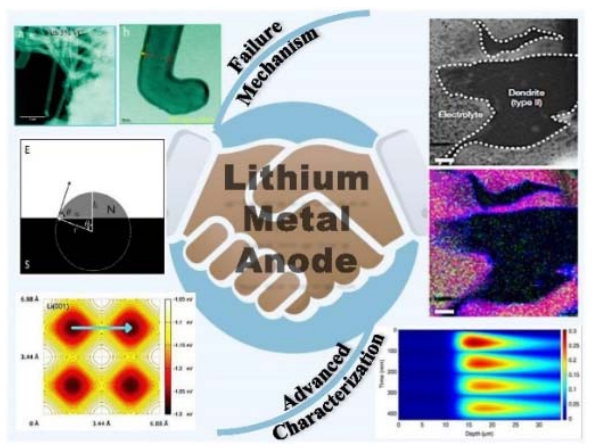
including low coulombic efficiency, poor cycle stability, and safety hazards. These problems associated with the Li metal anode are mainly caused by lithium dendrite growth, electrode volume changes, and interface instability. During the charge and discharge processes, $\mathrm{Li}$ deposition is not uniform across the electrode surface. Due to the low surface energy and high migration energy of Li metal, dendrites are preferentially formed during Li deposition. These dendrites proceed to grow with successive battery cycling, penetrate the separator, and eventually reach the cathode, thereby causing short circuits and thermal runaway. Additionally, the growth of the lithium dendrite is inherently correlated with the reaction interface structure, and dendrite growth results in inhomogeneity of the SEI (solid electrolyte interface) which is inevitably formed on the Li metal surfaces. Moreover, the volume change of lithium metal anodes is of importance, particularly during battery cycling and Li stripping/deposition processes which make the SEI layers considerably unstable. SEI layers usually cannot withstand the mechanical deformation caused by volume changes; such layers continuously break and repair during cycling and consume large amounts of the electrolyte. Additionally, some Li dendrites could break and become wrapped by SEI layers to form electrically isolated "dead" Li, which results in the loss of active $\mathrm{Li}$ in the Li metal anode. All these factors are responsible for the failure of Li metal anodes. Herein, recent investigations on the failure mechanisms of lithium metal anodes are reviewed and summarized, including the formation of SEI layers on the surface of Li metal anodes, the behavior and mechanism of lithium dendrite growth, and the mechanism of "dead" lithium formation. Additionally, some advanced characterization techniques for investigating lithium metal anodes are introduced, including in situ tools, cryoelectron microscopy, neutron depth analysis technology, and solid state nuclear magnetic resonance technology. These techniques enable researchers to gain in-depth insights into the failure mechanisms of Li metal anodes.
\end{abstract}

Key Words: Lithium battery; Lithium metal anode; Failure mechanism; Advanced characterization technology

\footnotetext{
Received: May 6, 2020; Revised: June 3, 2020; Accepted: June 4, 2020; Published online: June 10, 2020.

*Corresponding author. Email: ynzhou@fudan.edu.cn; Tel.: +86-21-65642685.

tThese authors contributed equally to this work.

The project was supported by the Science \& Technology Commission of Shanghai Municipality, China (19ZR1404200).

上海市自然科学基金(19ZR1404200)资助项目

(C) Editorial office of Acta Physico-Chimica Sinica
} 


\title{
金属锂负极失效机制及其先进表征技术
}

\author{
岳昕阳 $1, \dagger$, 马萃 $1, \dagger$, 包戬 1 , 杨思宇 ${ }^{2}$, 陈东 ${ }^{1}$, 吴晓京 ${ }^{1}$, 周永宁 1 , \\ 1 复旦大学材料科学系, 上海 200433 \\ 2 复旦大学化学系, 上海 200433
}

\begin{abstract}
摘要：尽管传统的石墨负极在商业化锂离子电池中取得了成功, 但其理论容量低 $\left(372 \mathrm{mAh} \cdot \mathrm{g}^{-1}\right)$ 、本身不含锂的先天缺陷 限制了其在下一代高比能量锂电池体系中的应用, 特别是在需要锂源的锂-硫和锂-空气电池体系中。金属锂因其极高的 理论比容量(3860 $\mathrm{mAh} \cdot \mathrm{g}^{-1}$ ) 和低氧化还原电势(相对于标准氢电极为 $-3.040 \mathrm{~V}$ ), 被认为是下一代锂电池负极材料的最佳 选择之一。但是, 金属锂负极存在库伦效率低、循环性能差、安全性差等一系列瓶领问题亟待解决, 而循环过程中锂枝 晶的生长、巨大的体积变化、以及电极界面不稳定等是导致这些问题的关键因素。本文综述了近年来关于金属锂负极瓶 颈问题及其机理, 包括金属锂电极表面固态电解质界面膜的形成, 锂枝晶的生长行为, 以及惰性死锂的形成。同时, 本 文还介绍了目前用于研究金属锂负极的先进表征技术，这些技术为研究人员深入认识金属锂负极的失效机制提供了重要 信息。
\end{abstract}

关键词：锂电池；金属锂负极；失效机制；先进表征技术 中图分类号: 0646

\section{1 引言}

随着移动电子设备、新能源汽车和分布式储 能系统的飞速发展, 人们对高能量密度 1 、长寿命 和高安全的锂离子电池的需求越来越紧迫。从电 池技术发展角度来看, 中国在电池制定标准上紧 跟日本和美国的脚步, 确定的目标是在2020年成 熟生产能量密度为 $300 \mathrm{Wh} \cdot \mathrm{kg}^{-1}$ 的一锂离子电池, 并 且在 2030 年将能量密度进一步提高到 500 $\mathrm{Wh} \cdot \mathrm{kg}^{-1}$ 。然而以目前的条件而言, 即使采用高比 能量的 $\mathrm{LiNi}_{0.8} \mathrm{Co}_{0.1} \mathrm{Mn}_{0.1} \mathrm{O}_{2}$ (NCM811)正极和石墨 负极, 锂离子电池的能量密度也只可接近 350 $\mathrm{Wh} \cdot \mathrm{kg}^{-1}$ 。如果希望将这一指标突破 $400 \mathrm{Wh} \cdot \mathrm{kg}^{-1}$, 甚至更高, 则需要一种具有全新正极和负极的锂 离子电池体系 ${ }^{2}$ 。研究表明, 锂-硫和锂-空气电池的 能量密度分别为 600 和 $900 \mathrm{Wh} \cdot \mathrm{kg}^{-13,4}$, 是传统锂离 子电池的数倍, 从而它们被认为是主导未来电池 系统发展的关键方向。然而, 这些新体系电池中的 正极不含有锂源, 因此传统的无锂负极材料, 例如 石墨、硅基材料等失去了直接应用的价值。

金属锂作为一种古老的锂源型负极材料, 在

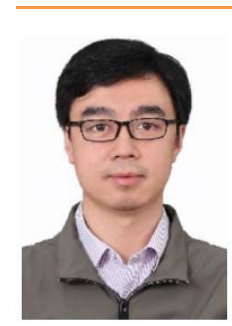

周永宁, 复旦大学青年研究员。2010年在 复旦大学材料科学系获得博士学位; 2010-2011年复旦大学化学系从事博士后 研究; 2012-2015年在美国布鲁克海文国 家实验室任助理研究员; 2015年回国加入 复旦大学材料科学系, 任青年研究员。主 要从事新型电池材料设计及机理研究。
离开人们视野近 50 年后因其极高的理论比容量 (3860 $\mathrm{mAh} \cdot \mathrm{g}^{-1}$ ) 和较低的氧化还原电势(相对于标 准氢电极为 $-3.040 \mathrm{~V}$ ) 而再次引起了人们的关注 ${ }^{5,6}$ 。 然而, 金属锂负极在电池循环过程时发生的枝晶 生长以及体积变化等问题严重阻碍了其实际应 用 $7-9$ 。一方面, 不断生长的锂枝晶会刺穿隔膜导致 电池内短路, 进而引发爆炸燃烧等安全危害 ${ }^{10}$ 。另 一方面, 枝晶的形成极大增加了电极的比表面积, 加剧了电极与电解液的副反应, 从而造成较高的 界面电阻以及容量损失 ${ }^{11,12}$ 。除此之外, 金属锂负 极在反复充放电过程中因溶解不均匀而脱落，导 致电极表面形成电绝缘的 “死锂” 层, 而这也是 造成电池性能衰退的主要原因之一13。

为提升金属锂电池的循环性能并克服以上安 全性问题, 进一步深入理解金属锂负极的失效过 程十分关键。金属锂电极在制备和保存过程中, 其 表面不可避免地存在少许杂质和结构缺陷, 因而 导致反应界面存在局部电流密度的不均匀。在锂 沉积初期, 锂倾向于在电流密度相对较高的活性 位点优先成核, 从而触发形成苔藓状沉积形貌, 并 随后导致锂枝晶的生长 ${ }^{11}$ 。在锂溶出过程中, 由于 新形成枝晶的活性高且阻抗低, 导致锂更倾向于 在枝晶部位优先发生溶解, 从而降低了枝晶与电 极主体之间的结合力。在体积变化引起的应力作 用下, 部分细弱枝晶会发生脱落并被固态电解质 界面(Solid electrolyte interface, SEI)膜包裹, 形成 电子绝缘的惰性 “死锂” 颗粒。随着循环的进行, 负极表面的死锂颗粒相互积压而形成致密的死锂 
层, 阻碍了锂离子的扩散和电子的传输, 从而导致 电极反应的极化升高。

因此, 深入认识金属锂表面SEI膜的特征和变 化, 锂枝晶的形成和生长机制, 以及 “死锂” 的形 成机制是有效抑制锂枝晶生长和提高金属锂电极 性能的关键, 同时也为寻找金属锂电极的解决方 案提供科学依据。然而, 由于金属锂化学活性高且 在大气气氛中十分不稳定, 许多传统的材料表征 手段在金属锂的相关研究中难以应用 14,15 。因此, 研究人员发展了一系列新的表征技术用于金属锂 电极的研究。

本文首先总结了金属锂电极的失效行为及相 关机理, 包括金属锂电极表面固态电解质界面 (SEI)膜的形成与特征, 锂枝晶的生长行为和机制, 以及 “死锂” 的形成机制和对电池性能的影响。同 时, 概述了近年来发展的用于金属锂电极研究的 先进表征技术，包括冷冻电镜技术、原位表征技 术、中子深度分析技术、固态核磁技术等, 以及利 用这些技术获得的研究成果。

\section{2 金属锂电极的失效机制}

\section{1 固态电解质界面(SEI)}

\subsubsection{SEI膜的形成机理}

由于有机溶剂的还原电位较低 $(<1.0 \mathrm{~V} v s$. $\mathrm{Li} / \mathrm{Li}^{+}$), 因此暴露在电解液中的负极表面在电流作 用下会直接与电解液成分发生不可逆的化学反 应, 这一表面反应过程一般在数毫秒内完成 ${ }^{16}$ 。该 反应形成的电极-电解质界面被称为固态电解质 界面(SEI)。如图 $1 \mathrm{a}, \mathrm{b}$ 所示, 负极材料 $\mathrm{Li}_{4} \mathrm{Ti}_{5} \mathrm{O}_{12}$ 和 多壁碳纳米管在嵌锂电位低于 $0 \mathrm{~V}$ 时的晶格体相 表面会生长一层具有一定厚度且属于无定型结构 的薄膜, 也就是 SEI膜, 且扫描电子显微镜(SEM)

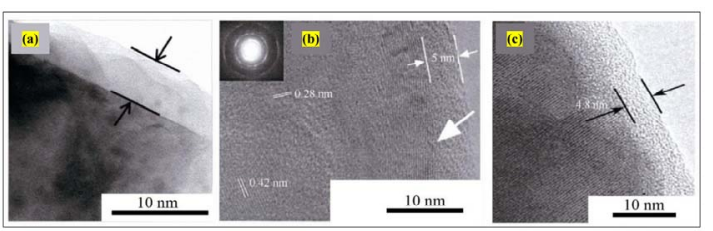

图 1 (a) $\mathrm{Li}_{4} \mathrm{Ti}_{5} \mathrm{O}_{12}$ 负极和(b)多壁碳纳米管负极 表面 SEI 膜的 TEM 照片; (c) $\mathrm{LiCOO}_{2}$ 正极循环 10 次后表面 SEI 膜的 TEM 照片 ${ }^{16}$

Fig. 1 TEM images of the SEI film onto the surface of (a) the $\mathrm{Li}_{4} \mathrm{Ti}_{5} \mathrm{O}_{12}$ and (b) the multi-walled carbon nanotube anode after discharging to below $0 \mathrm{~V}$;

(c) TEM image of the SEI film on the surface of the $\mathrm{LiCoO}_{2}$ cathode after 10 cycles ${ }^{16}$.

Adapted from Chemical Industry publisher.
照片显示的厚度为纳米级。此外, 正极表面也同样 会形成相应组分的 SEI 膜 (也被称为 Cathode Electrolyte Interface, CEI), 见图 $1 \mathrm{c}\left(\mathrm{LiCoO}_{2}\right.$ 表面形 成的SEI膜)。

德州大学奥斯丁分校的 Goodenough课题组 ${ }^{17}$ 系统建立了SEI膜形成与电解液性质之间的联系, 并提出了一种被普遍接受的SEI膜形成机理。如图 2所示, 阳极和阴极的电势分别为 $\mu_{\mathrm{A}}$ 和 $\mu_{\mathrm{C}}$, 最低未 占有轨道( LUMO) 和最高占有轨道(HOMO)的电压 分别为 $E_{\mathrm{LUMO}}$ 和 $E_{\mathrm{HOMO}}$ 。当阳极的电势 $\mu_{\mathrm{A}}>E_{\mathrm{LUMO}}$ 时, 阳极上的电子就会自发地转换到电解液的最 低未占有轨道上, 使得电解液被还原, 从而形成 SEI膜。同样, 当阴极电势 $\mu_{\mathrm{c}}<E_{\mathrm{HOMO}}$ 时, 电解液的 最高占有轨道上的电子会自发转换到阴极上, 从 而引发的氧化反应促使 SEI膜形成 ${ }^{17}$ 。由于SEI膜在 有机电解液中的溶解性极差, 且自身属于电子绝 缘体, 因此SEI膜在电池体系中能够稳定存在。一 方面, 对于锂嵌入-脱出型负极而言, SEI膜能有效 防止溶剂分子的共嵌入作用, 避免共嵌而导致的 负极材料结构佣塌, 因而大大提高了电极的循环 性能和使用寿命。另一方面, SEI膜作为致密的电 子绝缘膜能够避免电解液与负极活性物质直接接 触, 进而防止电极被电解液中的副产物深度刻蚀, 以减少电子从负极向电解液的转移, 以及溶剂分 子和电解质阴离子向负极的转移 18 。以六氟磷酸锂 $\left(\mathrm{LiPF}_{6}\right)$ / 碳酸甲乙酯 $(\mathrm{EMC})$ 电解液体系为例, 形成 SEI膜和副产物的反应方程式见反应式(1)和(2)。因 此, 稳定的SEI膜有利于提高电极结构的稳定性以 及电池的实际充放电容量。

$$
\begin{gathered}
2 \mathrm{CH}_{3} \mathrm{CH}_{2} \mathrm{OCO}_{2} \mathrm{CH}_{3}+2 \mathrm{e}^{-}+2 \mathrm{Li}^{+} \rightarrow \\
2 \mathrm{CH}_{3} \mathrm{CH}_{2} \mathrm{OCO}_{2} \mathrm{Li} \downarrow+\mathrm{C}_{2} \mathrm{H}_{6} \\
\mathrm{PF}_{6}^{-}+\mathrm{e}^{-}+2 \mathrm{Li}^{+} \rightarrow \mathrm{LiF} \downarrow+\mathrm{LiPF}_{5}
\end{gathered}
$$

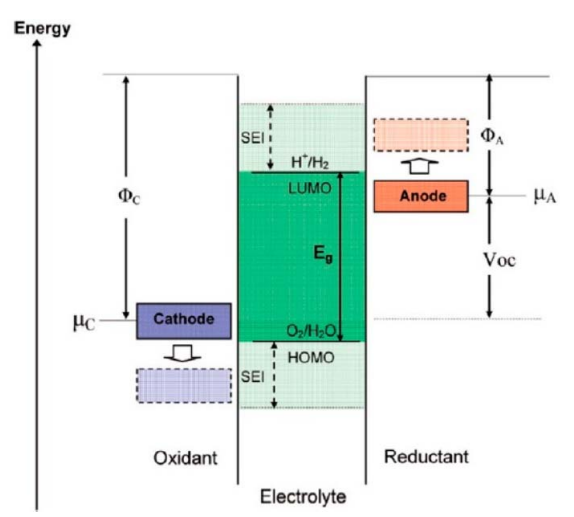

图 2 SEI 膜在液态电解液中的形成条件示意图 17

Fig. 2 Scheme of SEI formation condition in a liquid electrolyte ${ }^{17}$.

Adapted from American Chemical Society publisher. 
SEI膜对负极的电化学性能影响深远, 因而深 入了解SEI膜的形成机理以及结构特性是改善金 属锂和其他种类负极性能的有效途径。目前, 针对 于负极表面有五种SEI膜的结构解析被研究人员 广泛接受, 分别为Peled型、马赛克型、双层型、多 层型以及库伦反应型。

\subsubsection{Peled型SEI膜结构}

1979年, Peled ${ }^{19}$ 发现碱金属与非水电解液接 触时, 金属表面会形成一层厚度在1.5-2.5 nm左右 的异相膜, 并且这层膜具有电解质特点, 从而基于 这个现象首次提出了源自表面反应的SEI膜概念 (图3a)。在这种化学电池体系下, 金属电极表面的 电解质还原反应是分步进行的, 电解质中的某些 活性成分能够优先反应并沉积在电极表面, 导致 在整体SEI膜结构中存在一定量的Schottky缺陷, 作 为锂离子在 SEI膜中迁移的通道 ${ }^{20}$ 。

\subsubsection{2 马赛克型SEI膜结构}

1997年, Peled ${ }^{21}$ 通过阻抗测试发现SEI膜中的 微观颗粒相之间的晶界电阻 $R_{\mathrm{gb}}$ 要比体相的离子电 阻大, 说明了数个电解质的还原分解反应同时发 生在带有负电性的阳极表面, 从而形成了一个不 溶性的多相混合型沉积膜。这种不均匀的SEI结构 具有类似马赛克形貌(图3b), 因此定义为马赛克 型。其中, 多相物质的边界是锂离子传递的通道。

\subsubsection{3 双层型SEI膜结构}

2012年, Zhang等 ${ }^{22}$ 对厚度方向成分不均匀的 SEI膜进行了更加详细的研究, 并提出双层结构 SEI膜构型以及其离子传递机理(图3c)。他们认为 靠近金属锂负极表面的SEI膜是由低氧化态物质
构成, 例如 $\mathrm{Li}_{2} \mathrm{O} 、 \mathrm{Li}_{3} \mathrm{~N} 、 \mathrm{LiF} 、 \mathrm{LiOH}$ 及及 $\mathrm{Li}_{2} \mathrm{CO}_{3}$, 属于一种无机层。相反, SEI膜的外层结构由高氧 化态的物质所构成, 例如 $\mathrm{ROCO}_{2} \mathrm{Li} 、 \mathrm{ROLi}$ 以及 $\mathrm{RCOO}_{2} \mathrm{Li}$ ( $\mathrm{R}$ 为电解质包含的有机官能团), 属于一 种有机层。其中, 无机层可能是由最初沉积在电极 表面的有机成分随后转化形成的。这种双层结构 的SEI膜同时存在两种锂离子的扩散方式。有机层 为多孔结构而支持锂离子的液相扩散, 同时能够 帮助锂离子脱去溶剂化外壳。无机层属于固态离 子导体层, 从而锂离子通过相互碰撞的方式进行 迁移。

\subsubsection{4 多层型SEI膜结构}

1999年, Aurbach等 ${ }^{23}$ 通过对SEI膜进行红外光 谱、拉曼光谱以及X射线光电子能谱(XPS)测试研 究后首次提出具有多层结构的SEI膜。如图3d所 示, 以金属锂负极为例, 当新鲜的金属锂与电解质 溶液接触时, 电解液中包含的所有成分即开始还 原沉积在电极反应界面处, 且反应的选择性较低。 当形成一层电解质膜后, 其阻挡了电解液中某些 成分在电极表面的还原反应, 从而此时电解液分 解沉积反应的选择性升高。因此, 负极表面形成的 SEI膜属于多级层结构 22 。最后, 由于表面反应的 不确定性，导致在金属锂负极上形成的SEI膜同时 具有多层结构和马赛克型微观形貌。

\subsubsection{5 库伦反应型SEI膜结构}

1999年, Ein-Eli ${ }^{24}$ 首次提出了基于带电性质的 库伦反应型SEI膜结构, 具体可见图 $3 \mathrm{e}$ 。在这种 SEI 膜结构中, 沉积产物以正电性的锂离子为 “头”, 以带部分正电性的碳为 “脚”, 以负电性的氧为
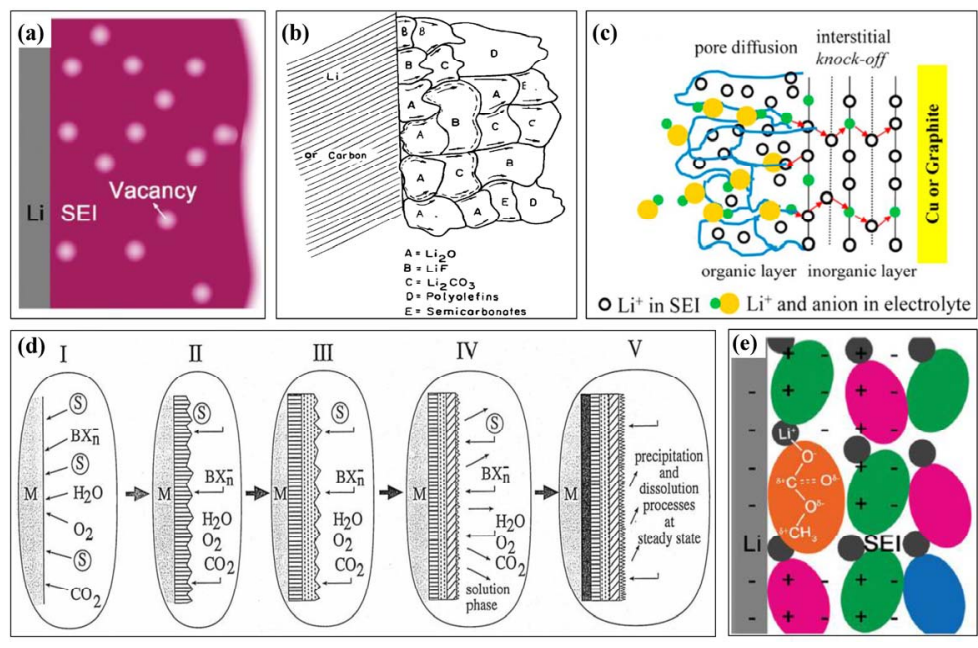

图 3 (a) Peled 型 ${ }^{19} 、$ (b) 马赛克型 ${ }^{21} 、$ (c) 双层型 ${ }^{22} 、\left(\right.$ d) 多层型 ${ }^{23}$ 以及(e) 库伦反应型 SEI 膜的结构示意图 ${ }^{24}$

Fig. 3 Schematic diagrams of (a) Peled SEI film ${ }^{19}$, (b) Mosaic SEI film ${ }^{21}$, (c) double-layer SEI film ${ }^{22}$, (d) multilayer SEI film ${ }^{23}$, and (e) coulomb-reaction SEI film ${ }^{24}$.

Adapted from American Chemical Society and Elsevier publishers. 
“尾”，呈平行于电极平面方向上线性规则排列。 这种双层结构的SEI膜特点在于其内部沉积物相互 连接, 而不是简单堆积。相比于马赛克型, 由于离 子对的静电作用, 库伦反应型SEI膜具有更好的结 构稳定性。

\subsubsection{SEI膜引发金属锂失效的机理}

SEI膜的物理化学性质对金属锂负极的影响 巨大, 且锂枝晶生长和死锂的形成也与 SEI膜的形 态息息相关。与其他种类负极不同, 无宿主的金属 锂负极在充放电过程中存在显著的界面结构演 变。因而当金属锂电极表面的SEI膜结构不稳定 时, SEI膜很容易在电极内应力的波动下发生破 裂, 从而暴露出新鲜的高活性金属锂界面。这些活 性界面会促使电解液还原而形成新的SEI膜, 从而 消耗更多的电解液, 严重情况下会使电解液干涸。 此外, 这些新裸露的活性区域导致相对较高的局 部电流密度, 会促进锂枝晶的生长, 进一步加剧界 面结构演变, 形成恶性循环。同时, 破裂的SEI膜 成分在电极循环过程中不断积累, 堆积在电极反 应界面, 阻碍了锂离子在电极界面的扩散, 增大了 电化学极化, 最终导致金属锂电极失效。SEI膜的 分布、组分、结构等因素皆会对锂的沉积行为产生 影响, 例如锂的成核过电位、成核位点分布、沉积 形貌结构等。因此, 对金属锂表面SEI膜的深入认 识, 是改善金属锂负极电化学性能的关键。

SEI膜总是由有机相和无机相组成, 其中无机 成分可以实现锂离子在SEI膜内的快速传输, 而有 机成分给予了 SEI膜柔软的特点以避免其在锂沉 积过程中发生破裂。总结大量文献可以得出, 金属 锂表面的SEI膜应具备以下特点: (1)物理化学性质 稳定, SEI膜在较宽的电压窗口下不溶于电解液; (2) 在SEI膜的厚度超过电子隧穿长度时表现完全的 电子绝缘性质; (3)具有高离子导电性, 锂离子通 过SEI膜时电阻小; (4)形貌结构稳定, 不随金属锂 电极的变化而改变; (5)与金属锂电极表面结合力 强, 在循环过程中不脱落粉化; (6)良好的力学性 能, 能够适应电极体积变化并阻碍枝晶穿刺; (7) SEI膜形成后不再参与电化学氧化还原反应。

\section{2 锂枝晶}

\subsection{1 锂枝晶的形成机理}

1970年, Whittingham ${ }^{25}$ 最早提出采用金属锂 作为负极材料, 并发明了首个可循环充放电的锂 电池。但是, 锂枝晶生长造成的安全性低、循环性 能差等问题, 导致金属锂负极在科学研究和工业 化道路上的发展受到了阻碍 26 。目前, 研究人员提 出了很多关于锂枝晶形成的机理以及其生长行为
的假设, 其中包含了成核/扩散模型、异相成核模 型、空间电荷模型以及SEI膜诱发成核模型等, 意 在为解决锂枝晶问题提供更加详细和坚固的理论 基础。

\subsubsection{1 成核/扩散模型}

首先, 由于金属锂的表面能低且锂原子在金 属锂表面受到的扩散壁垒高, 从而从热力学方面 来看, 枝晶在金属锂表面的形成与生长是一种本 质问题。这一点可以通过与镁金属对比来说明。通 过DFT计算发现在镁的密排六方结构中, $\mathrm{Mg}-\mathrm{Mg}$ 键的结合强度要高于体心立方结构的 $\mathrm{Li}-\mathrm{Li}$ 键。因 此, 镁在高维和低维相之间的自由能差高于锂, 可 见图 $4 a, b$ 。相对于金属锂沉积过程, 镁的沉积过 程更倾向于形成高维度结构 (二维和三维), 而锂的 沉积形貌更容易形成一维结构, 也就是刺状枝晶 形貌 27 。

另一方面, Groß等 28 通过理论模拟计算发现, 锂原子在 $\mathrm{Li}(001)$ 晶面上的扩散壁垒为 $0.14 \mathrm{eV}$, 而 镁原子在 $\mathrm{Mg}(0001)$ 晶面上的扩散壁垒为 $0.02 \mathrm{eV}$, 可 见图 $4 \mathrm{c}, \mathrm{d}$ 。一般来说, 原子在晶面处扩散地越快, 对应的沉积形貌就越平滑, 因为表面原子可以相 互连接以形成完整的表面层。相反, 当扩散壁垒较 大时, 原子在晶面扩散慢, 则得到的表面沉积结构 就相对粗粘。因为镁在镁晶体表面的扩散明显快 于锂在锂晶体表面的扩散, 从而镁的沉积路径更 加均匀且形貌更加平整, 而锂原子受到表面扩散 壁垒的限制, 往往集中在某一活性位点沉积, 从而 易于在电极表面形成一维枝晶形貌 ${ }^{28}$ 。

\subsubsection{2 异相成核模型}

当基底为非金属锂时, 例如铜和铁等, 锂离子 在其表面得到电子后发生的初期沉积过程被认为 是一种异相成核行为。由于锂沉积属于一种反应 过程, 从而它最终的沉积形貌受初期成核过程影 响较大 ${ }^{31}$ 。因此, 对锂的异相成核行为进行深入的 理解是控制锂沉积形貌的重点。Garcia等 ${ }^{29}$ 通过对 锂成核过程在热力学和动力学方面的模拟计算, 提 出了包含五种不同制度的异相成核模型, 分别为 抑制成核制度、长孕育时间制度、短孕育时间制 度、早期生长制度以及随后生长制度。如图 $5 \mathrm{a}, \mathrm{b}$ 所示, 首先是抑制成核制度。期间锂的晶胚处于热 力学不稳定状态, 因而倾向于重新溶解进入电解 液中。在长孕育时间制度中, 锂晶胚的吉布斯自由 能已经克服了成核的临界能量屏障, 但推动其继 续生长并超过临界成核尺寸的驱动力仍与热波动 相当。因此, 锂晶胚处于一种亚稳态, 晶胚的生长 与溶解同时存在, 导致一段时间内不发生有效生 


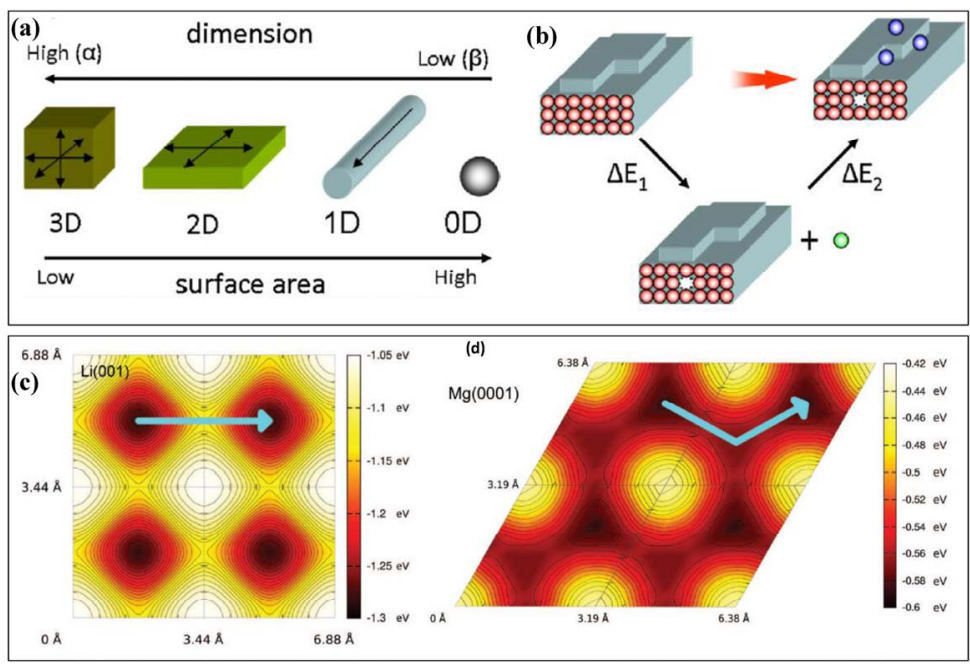

图 4 (a)高维相位和低维相位示意图阶段 ${ }^{27}$; (b) 原子从体相向表面移动 ${ }^{27}$; (c) $\mathrm{Li}$ 原子在 $\mathrm{Li}(001)$ 晶面上的势能面和 (d) $\mathrm{Mg}$ 原子在 $\mathrm{Mg}(0001)$ 晶面上的势能面 ${ }^{28}$

Fig. 4 (a) Schematic of high-dimensional and low-dimensional phases ${ }^{27}$; (b) movement of one atom from the bulk phase to the surface ${ }^{27}$; potential energy surfaces of (c) a $\mathrm{Li}$ adatom on $\mathrm{Li}(001)$ and (d) a $\mathrm{Mg}$ adatom on $\mathrm{Mg}(0001){ }^{28}$. The contour spacing in (c) is $0.05 \mathrm{eV}$ while it is $0.02 \mathrm{eV}$ in (d). Adapted from Elsevier and American Institute of Physics publishers.
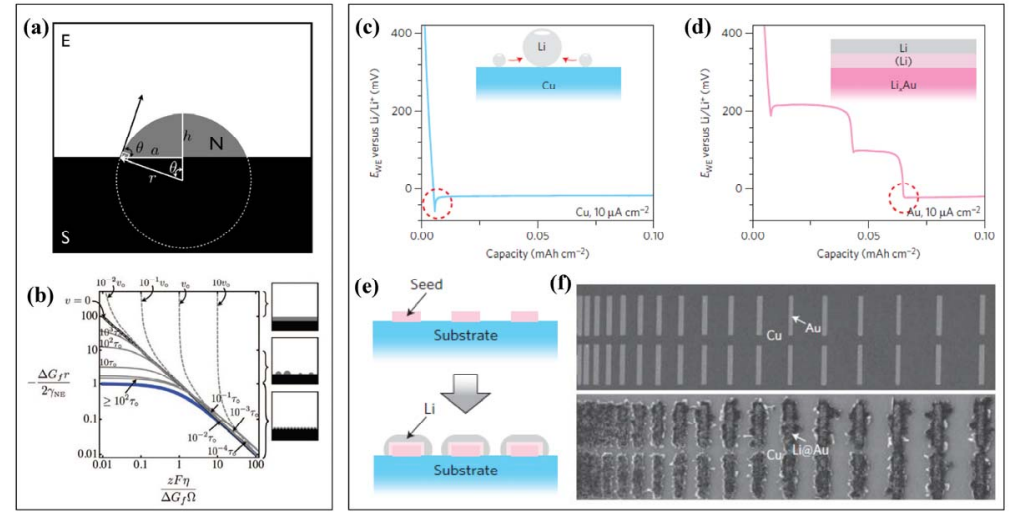

图 5 (a)在平整集流体上沉积球帽型锂晶核过程的示意图，锂成核初期和锂枝晶生长的行为 ${ }^{29}$;

(b) 电流密度为 $10 \mu \mathrm{A} \cdot \mathrm{cm}^{-2}$ 时锂在铜和金集流体表面沉积过程的电压-容量曲线 ${ }^{29}$; (c)锂在两相介质上的沉积示意图 (蓝色为 $\mathrm{Cu}$, 粉色为 $\mathrm{Au}$ ) 以及锂在 $\mathrm{Cu} / \mathrm{Au}$ 集流体上沉积前后的 SEM 图 ${ }^{30}$

Fig. 5 (a) Scheme of a spherical cap-shaped nucleus plated on a flat substrate immersed in an electrolyte, and regimes of behavior during the initial stages of nucleation and growth of Li dendrites ${ }^{29}$; (b) voltage profiles of galvanostatic $\mathrm{Li}$ deposition on a copper and gold substrate at $10 \mu \mathrm{A} \cdot \mathrm{cm}^{-2}{ }^{29}$; (c) schematics of patterned Li deposition (Cu: blue, Au: pink) and SEM images of a gold strip array with various separations before (top) and after (bottom) Li deposition ${ }^{30}$. Adapted from Electrochemical Society and Springer Nature publishers.

长。提高沉积过电势后进入短孕育时间制度, 这时 锂的临界成核热力学半径和动力学临界半径非常 接近, 有利于晶胚加速成长。此外, 由于总能量屏 障很小, 高过电位(短孕育时间制度)有利于锂晶胚 的窄尺寸分布。最后, 在早期和随后的生长制度 中, 热力学和动力学稳定的锂晶核生长到相同的 终端尺寸, 且一旦锂晶核稳定存在, 则对应的生长 速率便保持恒一, 直到反应结束。
斯坦福大学的Cui课题组 ${ }^{30}$ 直观对比了锂在不 同基体上的异相成核生长过程。如图 $5 \mathrm{c}$ 所示, 当表 面光滑的铜片作为集流体时, 初期锂沉积的电压容量曲线出现一个明显的电压降信号, 随后电压 回升并呈现稳定的平台。这里, 电压下降的尖端值 相对于电压平台值的差可认为是锂沉积过程中的 初期成核过电位 ${ }^{32}$ 。由于锂和铜之间存在较大的热 力学不匹配, 因此大约 $40 \mathrm{mV}$ 的过电位用来克服异 
相成核的障碍。相反, 当采用金集流体时, 在 $0 \mathrm{~V}$ 以上出现了两个锂化电压平台, 对应了 $\mathrm{Li}-\mathrm{Au}$ 合金 的形成。之后, 在 $0 \mathrm{~V}$ 以下, 锂在金基体表面沉积 的电压-容量曲线显示出拐角信号, 这与铜集流体 形成了鲜明的对比。说明, 锂在 $\mathrm{Li}-\mathrm{Au}$ 合金上初期 成核所需要的过电位几乎为零。SEM表征可以更 加直观的发现, 在限定锂沉积的容量下, 相对于铜 集流体锂更倾向于在金表面进行沉积。综上可知, 通过对集流体进行相应的修饰以调控锂的异相成 核过程，可以实现金属锂的定位生长与均匀沉积。

\subsubsection{3空间电荷模型}

空间电荷模型是一种被广泛接受的锂枝晶成 核理论。1990年, Chazalviel等 ${ }^{33}$ 证明了枝晶的产生 主要是由于稀溶液中形成的空间电荷层所引起。在 如图6a所示的金属锂对称模拟电池中, 当电解液 浓度较低时, 电极表面的锂离子得到电子后被不 断还原, 而对流区的锂离子向沉积界面进行传质 扩散需要一定时间, 从而形成锂离子浓度梯度。因 此, 电极沉积界面区域的锂离子浓度逐渐减小而 打破了电中性规则, 诱发在部分电极表面区域形 成了大量的空间电荷, 可见图6b所示的区域II ${ }^{34}$ 。 由于锂离子扩散受电场迁移控制, 从而空间电荷 导致电极表面的锂离子流分布不均匀, 这促进了 锂枝晶的形成。当在稀盐溶液中且电流密度较大
的条件下, 空间电荷 $Z_{\mathrm{c}} e C_{\mathrm{c}}$ 的存在将促使具有分支 形貌的金属锂沉积层形成与生长 ${ }^{35}$ 。另一方面, 基 于空间电荷模型的枝晶生长初始时间 $(\tau)$ 称为 Sand 时间, 计算公式如公式(3):

$$
\tau=\pi D \frac{e C_{0}\left(\mu_{\mathrm{a}}+\mu_{\mathrm{Li}^{+}}\right)^{2}}{2 J \mu_{\mathrm{a}}{ }^{2}}
$$

其中 $D$ 为双极扩散系数, $e$ 为电荷, $C_{0}$ 为电解液初 始浓度, $J$ 为有效电流密度, $\mu_{\mathrm{a}}$ 和 $\mu_{\mathrm{Li}^{+}}{ }^{+}$分别为阴离子 和 $\mathrm{Li}^{+}$的迁移率 ${ }^{35}$ 。

当Sand时间较短时, 空间电荷现象引发的锂 枝晶生长容易发生, 并且随着沉积容量的增长, 电 极表面的锂枝晶层逐渐向电极对流区扩散(对电 极区域), 如图6c所示。当发生锂枝晶刺穿隔膜时, 如图6d所示, 平稳的电压曲线突然发生较大的波动 (跳水现象), 对应电池体系内短路的发生。Bazant 等 ${ }^{36}$ 通过实验证实了 Sand时间模型。当电流密度较 大时, 则 $\tau$ 越小, 表明电池发生内短路所需的时间 越少, 如图6e, f所示。因此, 通过降低有效电流 密度或加速锂离子迁移率可以延长Sand时间, 即 延缓锂枝晶在电极反应界面区域的产生 ${ }^{35}$, 提高电 池的循环寿命和安全性。

\subsubsection{SEI膜诱发成核模型}

在电池体系中，金属锂表面自发形成的SEI膜 对锂沉积形貌有着非常深远的影响。一方面, 在循
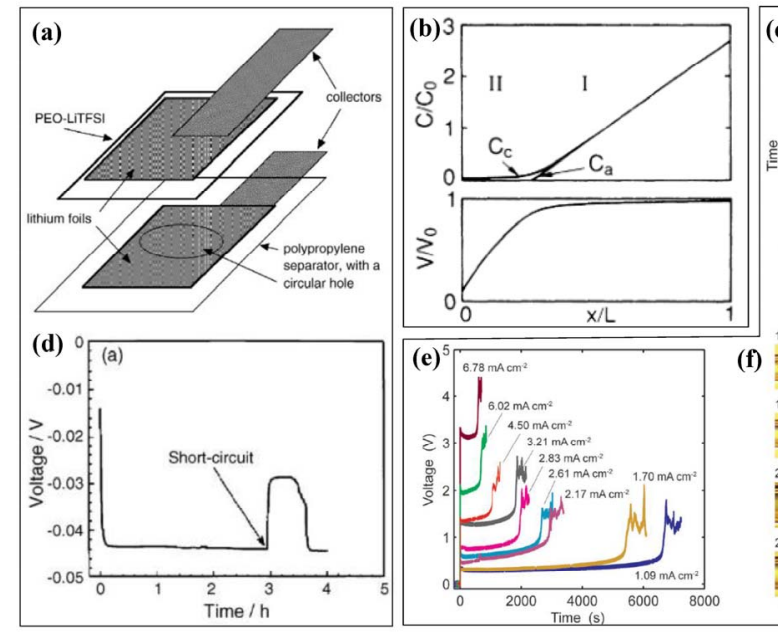

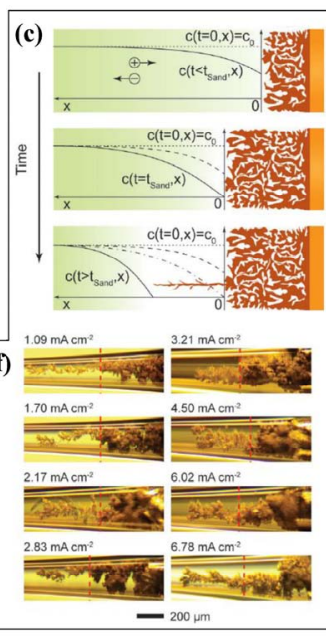

图 6 (a) 对称电池示意图 ${ }^{35}$; (b) 从均质沉积假想模型的数值模拟中获得的离子浓度与静电势的图形 ${ }^{34}$;

(c)浓差极化过程中电沉积锂枝晶生长的机理示意图 ${ }^{36}$; (d) 对称电池发生短路时的电压曲线图 ${ }^{35}$;

(e) 不同沉积电流密度下的毛细管模拟电池的电压曲线和(f)锂枝晶的生长状态 ${ }^{36}$

Fig. 6 (a) Schematic of symmetrical battery ${ }^{35}$; (b) profiles of ion concentration and electrostatic potential resulting from numerical simulation in the hypothetical case of uniform deposition with negligible growth of the cathode ${ }^{34}$; (c) mechanism of lithium dendrite growth with the change of concentration polarization ${ }^{36}$; (d) voltage curve diagram when short circuit occurs ${ }^{35}$; (e) voltage curve of capillary simulated battery under different deposition current densities, and (f) lithium dendrite growth state ${ }^{36}$. 
环过程中, 金属锂的体积变化效应诱发电极界面 产生严重的应力波动, 导致SEI膜结构破裂, 并暴 露出高活性的金属锂表面, 进而引发不均匀的锂 沉积行为以及促进刺状锂枝晶生长。另一方面, 假 设SEI膜结构完整, 但它的内在本质(物化性质)也 同样会显著地影响锂的沉积过程。Unocic等 ${ }^{37}$ 通过 原位电化学透射电子显微镜技术对电极表面的锂 沉积反应进行了详细观察。如图7a可见, 在相对高 的电势下, 电极表面的电解质发生分解并沉积在 电极的表面(1: 黑色电极与虚线之间的形貌对应 无定形的SEI膜)。此时, 由于SEI膜的电子电导性 较差, 锂离子需要扩散通过SEI膜并吸附在电极表 面进行沉积。随着电势进一步降低, 可见图7a中的 步骤3, 在SEI膜与电极之间形成了块状的锂沉积 物(红色箭头指向)。然而, 在整个沉积过程中, SEI 膜的生长一直存在, 其结构几乎不会维持稳定, 它 们会持续地增厚并全程自我更新。

$\mathrm{Li}$ 等 38 通过原位环境透射电镜技术对锂枝晶 的形成进行了实时监测。如图 7b所示, 锂首先在电 极表面成核且形成零维结构的球形沉积形貌。沉
积 $50 \mathrm{~s}$ 后, 初期球形的锂生长成具有一维结构的棒 状锂(晶须)。6 $5 \mathrm{~s}$ 后, 锂沉积的生长方向发生了明 显改变, 且棒状锂分解成两个在不同生长方向上 的锂沉积部分, 图中 $\wedge 1$ 和 $\wedge 2$ 。 $80 \mathrm{~s}$ 后, 锂沉积物 的扭曲程度变大, 形成分支枝晶结构。这说明 SEI 膜对锂的沉积行为以及形貌有一定的导向作用 39 。 可见图 $7 \mathrm{c}$, 枝晶的生长可分解为四步。第一阶段, 球形金属锂核心在电极表面形成，其直径与时间 的平方根成正比, 表明沉积行为受到锂离子扩散 的限制, 这与通过SEI膜的短程固态传输相关, 而 与长程液态扩散无关。同时, SEI膜使锂沉积物表 面钝化, 并逐渐降低锂沉积速率 40 。第二阶段, 继 续沉积的锂将最初形成的球体推离电极, 说明新 的沉积发生在 “晶须” 的根部。这是因为SEI膜的 存在一定程度上抑制了锂沉积, 导致沉积物根部 形成应力场并逐渐积累 (图7d), 从而在应力释放的 作用下, 锂更倾向于在根部进行沉积。同时, 由于 这种根部生长模式, 使得根部区域的SEI膜比顶部 区域的SEI膜更薄, 因此根部区域的界面阻值更低, 其更容易发生锂离子的还原反应 ${ }^{41}$ 。此外, 在这个
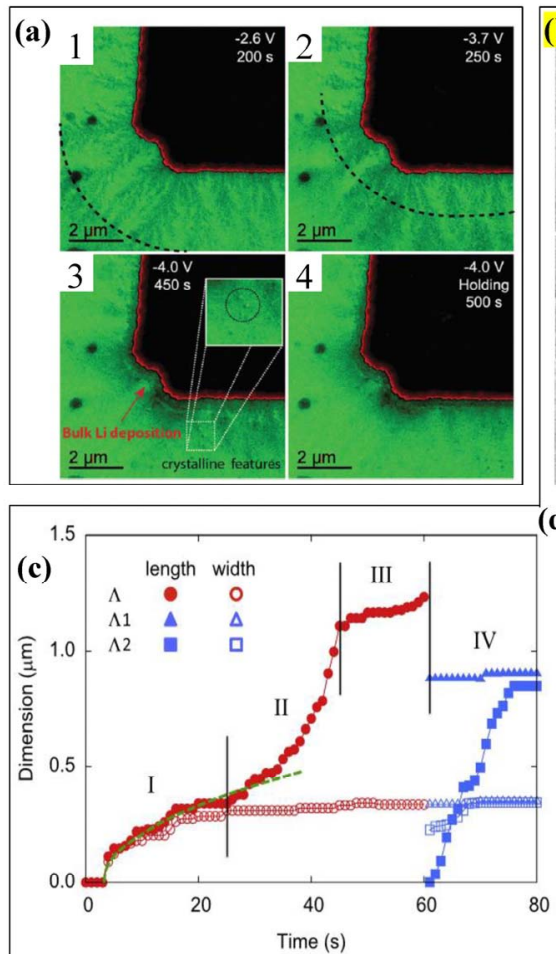

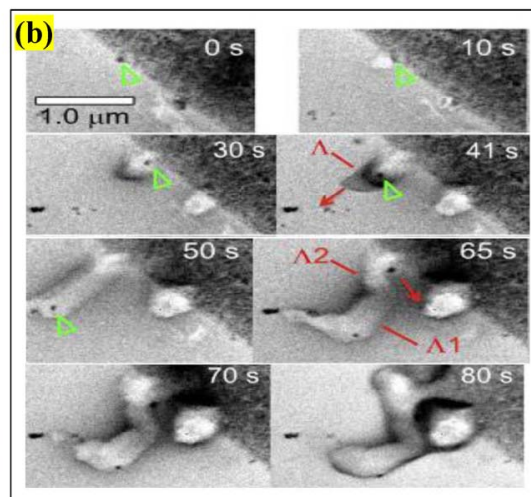

(d) Stage 1: Hindered surface
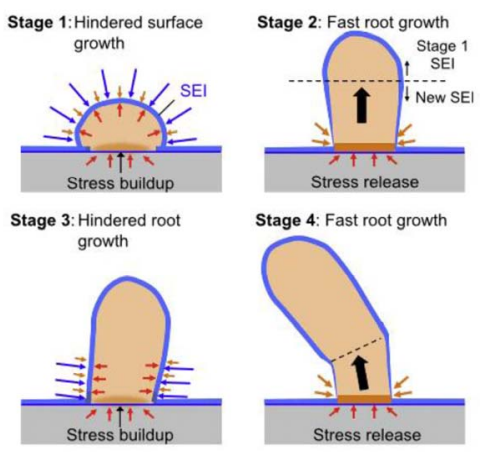

图 7 (a)原位电化学透射电子显微镜对锂离子在金电极上沉积过程的观察 ${ }^{37}$; (b) 原位环境透射电镜对

锂枝晶生长的观察; (c)应锂晶须长度和宽度的变化; (d)锂枝晶的根部生长与扭结形成示意图 ${ }^{38}$

Fig. 7 (a) The wave front corresponds to amorphization of SEI dendrites by Li deposition as is highlighted ${ }^{37}$;

(b) in situ TEM observation of a single Li whisker growth under $-6.0 \mathrm{~V}$ vs. LCO biasing; (c) change in the length and the width of the lithium whisker; (d) schematic illustration explaining root growth mechanism of lithium whiskers ${ }^{38}$. 
阶段观察到的晶须长度生长迅速, 而其宽度几乎 保持不变。第三阶段, 锂生长速率显著降低, 这可 能是因为在新形成的晶须上(根部), SEI膜的厚度 逐渐增长且结构趋近完整, 导致界面电阻提高以 降低锂沉积的选择性。此时, 如同第一阶段和第二 阶段, 熟化的SEI膜抑制了正常锂沉积的进行, 因 此沉积物根部再次积攒应力。第四阶段, 沉积物根 部压力释放, 扭结(拐点)开始形成在棒状理的晶须 上, 并把它分为了两部分 $(\wedge 1$ 和 $\wedge 2)$, 见图 $7 \mathrm{c}, \mathrm{d}$ 。 新形成的片段 $\wedge 2$ 从根部生长并将晶须的原有部 分推向不同于原晶须的生长方向, 并且 $\wedge 2$ 在长度 和宽度上有所增长, 而 $\wedge 1$ 的长度基本保持不变。

除了锂成核之外, 还有很多因素对金属锂的 沉积行为产生影响, 例如自生温度、电流密度以及 电场等 ${ }^{42}$ 。Hong等 ${ }^{43}$ 认为电池体系的自生热与电化 学反应势垒和锂离子扩散势垒有关, 其中, 沉积过 程中较大的电化学反应势垒会加速锂枝晶的生 成, 而较大的锂离子扩散势垒则会减缓枝晶的生 成速率。他们发现在较高的过电位条件下, 反应的 自生温度可达到 $350 \mathrm{~K}$, 且锂离子迁移率随着温度 的升高而提升数倍, 因而缓解了电极反应界面处 的锂离子浓度极化现象, 降低了锂枝晶的生长趋 势。另一方面, 从初期锂成核方面来看, 除了锂离 子流的分布影响外, 电极反应界面的感应电场对 锂枝晶的生长演化也起到了重要的引导作用。在 传统平面反应界面中, 例如二维的金属锂䈃, 其内 部形成的电场为垂直于隔膜方向分布, 因而在电 场的驱动下, 锂枝晶主要沿着垂直于隔膜的方向 进行生长, 并直接对隔膜形成威胁。Yang课题组 ${ }^{44}$ 从电场的角度出发, 设计了一种 “蛇笼” 状阵列 结构, 通过扭曲微观电场分布以诱导锂枝晶沿水 平方向的阵列生长, 从而提高锂金属电池的循环 性能与安全性能。他们发现具有微孔阵列结构的 铜集流体可实现平行于隔膜方向的电场分布, 且 在这样的电场力作用下, 锂枝晶会沿着平行于隔 膜的方向生长, 从而降低了枝晶生长对隔膜产生 的应力。

\subsection{2 枝晶引发金属锂电极失效机制}

上述不同的锂枝晶形成机理皆表明, 随着金 属锂电极循环, 以及沉积容量的提高, 在电极反应 界面形成的锂枝晶, 其尺寸会随之增长。当枝晶生 长到一定尺寸后, 一方面会刺穿隔膜, 造成电池内 短路并引发热失控。另一方面, 锂枝晶的生长增加 了电极反应界面的比表面积, 从而加剧了副反应 并持续消耗电解液, 最终导致电解液干涸使得电 池失效。此外, 枝晶的生长也加速了惰性 “死锂”
的出现。导致了金属锂负极的库伦效率降低, 并极 大降低了金属锂的利用率。

\section{3 锂金属体积变化}

锂离子电池负极材料在充放电过程中皆伴随 一定程度的体积变化效应。石墨与锂离子发生插 层化反应形成锂-石墨层间化合物对应的体积变 化较小 $(10 \%)$, 因此电极结构稳定, 长循环性能优 异 ${ }^{4}$ 。硅负极与锂离子可发生合金化反应形成 $\mathrm{Li}_{22} \mathrm{Si}_{5}$, 对应的理论容量巨大 $\left(4200 \mathrm{mAh} \cdot \mathrm{g}^{-1}\right)$, 并 且体积变化超过 $400 \%$, 是石墨材料的数十倍之多。 因此硅负极的循环性能较差 ${ }^{46}$ 。金属锂作为一种无 宿主活性材料, 在充放电过程中随着锂的溶出和 沉积会产生显著的体积变化, 因此电极的界面也 始终处于动态过程。当前商用石墨负极的面容量 至少为 $3 \mathrm{mAh} \cdot \mathrm{cm}^{-2}$, 相当于厚度为 $14.6 \mu \mathrm{m}$ 的活性 金属锂所提供的容量。也就是说, 当采用金属锂负 极时, 其在循环过程中由于体积变化引起的界面 移动可能超过几十微米, 这给SEI膜的稳定性带来 巨大的挑战 6 。另一方面, 伴随锂枝晶生长, 锂的 再沉积形貌往往为疏松多孔，且表面粗粘度上升。 这导致沉积后的金属锂负极厚度相对提高, 并且 随着循环的进行不断增长, 造成体系内部的锂沉 积环境发生改变而促使电池的失效(电解液干涸、锂 枝晶生长、界面电阻增大等)。本课题组前期研究 发现, 当采用传统的锂片电极时(厚度为 $420 \mu \mathrm{m}$ ), 在碳酸盐类电解液体系中沉积 $20 \mathrm{mAh} \cdot \mathrm{cm}^{-2}$ 后的 电极厚度增加了 $280 \mu \mathrm{m}$, 达到 $700 \mu \mathrm{m}$, 对应的体 积变化率为 $66.67 \%$ 。然而沉积 $20 \mathrm{mAh} \cdot \mathrm{cm}^{-2}$ 的锂理 论上只应增长 $97 \mu \mathrm{m}$, 表明再沉积锂具有更低的堆 积密度。

\subsection{1 死锂层的形成原因}

金属锂电极表面死锂层的形成与电极的体积 变化有关 ${ }^{47}$ 。如图 $8 \mathrm{a}$ 所示, 沉积过程中, 电极表面 由于不均匀的成核与生长过程而倾向于形成具有 苔藓状的锂沉积形貌。初期脱锂时, 锂优先从先前 存在的苔藓状锂中剥离出来。这是因为相比于电 极主体中的块状锂, 苔藓状锂的活性更高, 从而表 现出更低的阻抗特征。随着锂脱出进行, 电极表面 苔藓状锂的尺寸进一步减小, 导致这些部位的阻 抗变大。由于体积变化的存在, 这些小尺寸的苔藓 状锂在应力的驱使下将会与电极主体机械分离, 从 而形成完全电子绝缘的惰性颗粒- - 死锂(表面 被SEI膜包裹), 并沉积在电极反应界面处 ${ }^{11}$ 。接下 来, 锂主要从块状锂中脱出, 使得电极表面形成大 量的小洞, 而这些位置具有较强的活性可作为锂 成核生长的热点而造成新的苔藓状锂形成。此外, 


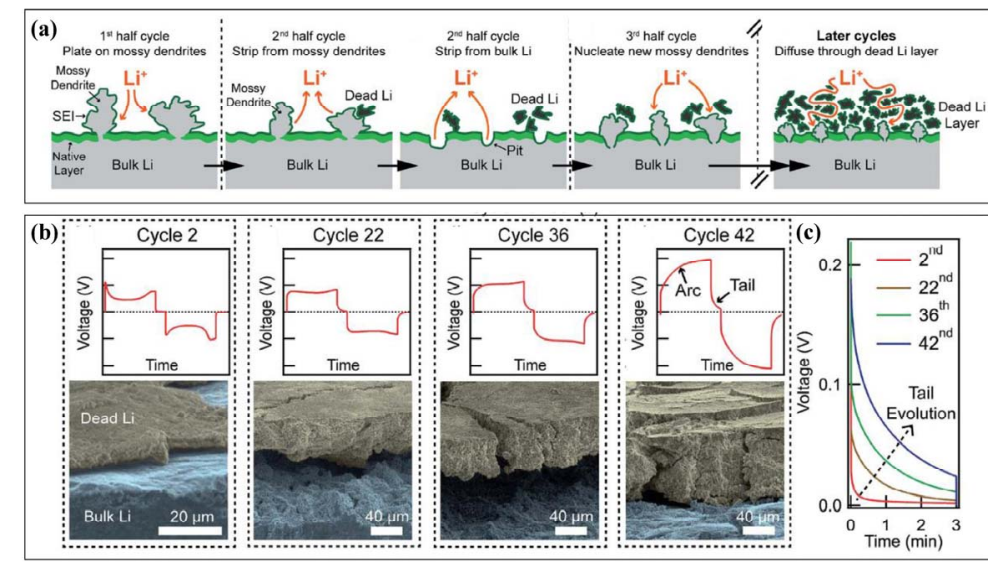

图 8 (a)金属锂负极表面死锂层的形成机锂示意图; (b) 电极表面死锂层的厚度与电极嵌脱锂电压曲线之间的关系;

(c)电极表面死锂层的厚度与电极嵌脱锂电压曲线尾部特征之间的关系 47

Fig. 8 (a) Schematic diagram of the dead $\mathrm{Li}$ layer formation onto the Li metal anode surface;

(b) relationship between the thickness of the dead Li layer and the voltage curve of the Li intercalation;

(c) cycle-to-cycle evolution of voltage tails ${ }^{47}$.

Adapted from Elsevier publisher.

由于硬壳电池的紧装结构, 电极表面形成的死锂 受到较大的机械压缩应力, 从而电极界面易形成 一层致密的惰性死锂层 ${ }^{13}$ 。

\subsection{2 死锂层对电化学性能影响}

死锂层的存在很大程度上阻碍了锂离子在电 极表面的扩散, 这一方面促进了锂枝晶的形成, 另 一方面提高了电化学反应极化。在长循环后, 死锂 的增长导致活性锂减少, 同时 SEI层的厚度和界面 电阻急剧增加, 导致电池失效。Dasgupta等 ${ }^{48}$ 系统 研究了金属锂电极表面死锂层对电化学性能的影 响。可见图 $8 \mathrm{~b}$ 和 $\mathrm{c}$, 随着首次循环的进行, 电极表 面死锂层的厚度逐渐增加, 并且对应的电压曲线 也发生了变化。在第二次循环时, 电压曲线呈现出 明显的 “头尾高一中间低” 的形状, 反应了锂在电 极表面成核与生长过程的不同环境。当循环至 36 次时, 死锂层的厚度超过 $50 \mu \mathrm{m}$, 并且电压曲线相 对之前发生了较大变化, 初始电压曲线的 “尖” 变成了 “弧”。并且, 进一步循环至42次, “弧” 型电压曲线特征更加明显, 说明电极反应的极化 变大。此外, 如图8c所示, 随着循环继续进行, 电 压曲线中与平衡浓度分布松别相关的尾部曲线变 得逐渐明显, 表明电池体系中与质量传输联系的 过电位逐渐变大。这主要是因为致密的惰性死锂 层阻碍了电解液中锂离子的有效扩散, 增大了电 极的浓度极化现象 ${ }^{49}$ 。

\section{3 先进表征技术在金属锂电极中的应用}

\section{1 原位表征技术}

采用传统非原位表征技术只能得到金属锂电 极和界面在稳定状态下的结构、形貌和化学组成 等信息, 而实时监测和跟踪循环过程中金属锂电 极的演变对深入理解金属锂电极的电化学性能十 分重要。因此, 原位表征技术受到了研究人员的青 睐。本节将介绍原位光学显微镜、电子显微镜、原 子力显微镜、 $X$ 射线衍射、 $X$ 射线光电子能谱、拉 曼光谱等技术在金属锂电池研究中的应用。

\subsection{1 原位光学显微镜技术}

随着金属锂电极研究的深入和表征技术的发 展, 原位电子显微镜、原子力显微镜等高端显微镜 越来越多地应用在金属锂负极的研究中，这些技 术虽然可以实现纳米尺度的观测, 但是设备昂贵、 对样品要求高、表征耗时费力、观测范围较小, 原 位装置复杂, 并容易对样品造成损伤。原位光学显 微镜技术虽然放大倍率相对较低, 只能在微米、毫 米级尺度宏观观察样品形貌, 但是相比于原位电 子显微镜等技术, 它具有设备经济简单, 测试便捷 易操作, 对样品无特殊要求等特点, 而且可以实现 对样品的大范围观测, 具有很大的实用性。近年 来, 原位光学显微镜技术已为金属锂电池形貌大 范围的实时跟踪提供了解决方案, 深化了人们对 金属锂表面宏观形貌变化规律的认识。

Zhou课题组 ${ }^{50}$ 使用光学显微镜和电池测试平 台搭建了一种简易的原位测试装置(图9a), 并观察 了两种金属锂复合电极的实时形貌变化。可见图 $9 \mathrm{~b}$, 基于碳毡的骨架的金属锂复合电极 ( $\mathrm{CFCu}-\mathrm{Li}$ ) 在循环了 120 次后电极与隔膜的接触依然紧密, 除 


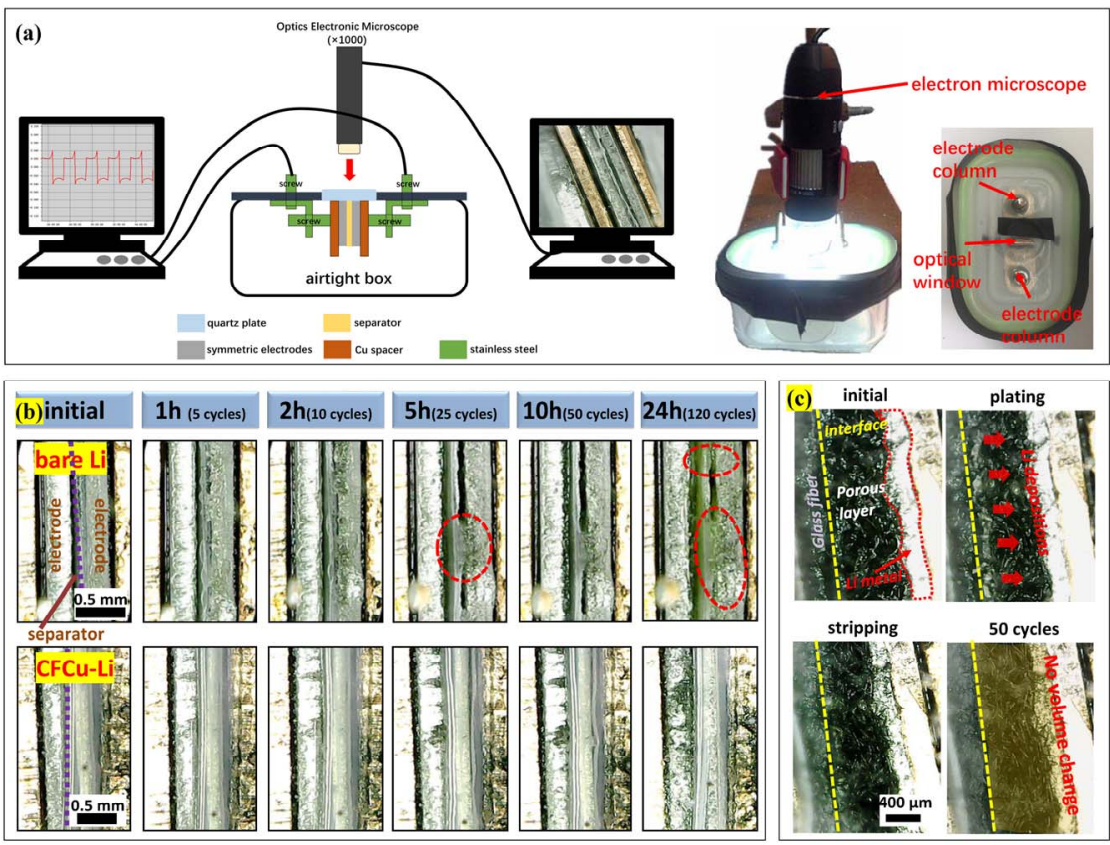

图 9 (a) 原位光学显微镜装置示意图; (b) 对锂片电极与 CFCu-Li 负极原位光学显微镜图 ${ }^{50}$;

(c) CFZO-Li 负极原位光学显微镜图 51

Fig. 9 (a) Schematic diagram of the in situ optical microscopy ; (b) in situ optical microscopy images of CFCu-Li anode ${ }^{50}$; (c) in situ optical microscopy images of CFZO-Li anode ${ }^{51}$.

Adapted from Elsevier publisher.

了电极界面发生了部分氧化以外, 电极的厚度没 有发生较大变化。而对锂片电极对照组锂枝晶生 长和死锂形成十分明显, 循环120次后, 除了原始 区域的锂枝晶结构外，在电极上方也形成了枝晶 形貌, 同时电极的体积发生了较大的变化。这说明 锂的沉积/溶解行为在 $\mathrm{CFCu}$ 骨架的参与下得到了 调控, 从而锂枝晶和死锂的形成得到了抑制。Yue 等 ${ }^{51}$ 设计了一种具有花瓣状 $\mathrm{ZnO}$ 修饰层的 $3 \mathrm{D}$ 碳毡 骨架(CFZO), 并通过控制锂注入时间成功制备得 到具有两层结构的复合金属锂负极(CFZO-Li)。原 位光学显微镜实时观测到了电极的形貌变化, 可 见图9c。初始电极的富锂致密层和贫锂多孔层可 以在横截面照片中清晰地区分开。放电后, 发现锂 主要沉积在电极表面的多孔层中(红色箭头指示)。 50 次循环后电极仍维持恒定的厚度变化, 这说明 CF骨架能够抑制金属锂在重复充放电过程中的体 积效应以提高电极结构的稳定性。

$\mathrm{Li}$ 等 ${ }^{52}$ 也应用原位光学显微镜技术实现了对 锂枝晶生长的实时观察, 并提出了一种与传统观 念相反的枝晶演化机制。可见图 $10 \mathrm{a}$, 当电流密度 较小时, 电极界面形貌随着循环的进行逐渐变得 粗糙且形成了大量的锂枝晶形貌, 而在电流密度 较大时电极的形貌演变相对平缓。在图 $10 \mathrm{~b}$ 中, $\mathrm{Wu}$ 等 53 通过该技术观察到 $3 \mathrm{D}$ 亲锂性 $\mathrm{LiB}$ 骨架上原位
生成的的 $\mathrm{Mg}$ 掺杂 $\mathrm{Li}-\mathrm{LiB}$ 合金层能够抑制枝晶生长 和体积膨胀。Zhang课题组 ${ }^{54}$ 调控液态电解液中锂 离子溶剂化层的组成和结构, 改善SEI和锂沉积的 均匀性, 从而增强了锂金属电池在液态电解液中 的循环稳定性。可见图 $10 \mathrm{c}$, 原位光学显微镜图像 显示相比于对照组 $\mathrm{FEC} / \mathrm{LiNO}_{3}$ 电解液中具有更加 均匀稳定的锂沉积形貌。Wang课题组 ${ }^{7}$ 研究了金属 锂在 $\mathrm{Li}_{x} \mathrm{Si}$ 载体上进行沉积剥离的电化学性质, 并通 过该技术观察了对照组在循环过程中的形貌宏观 变化。图10d显示在初始沉积过程中对称电池金属 锂表面会形成山丘状的沉积突起, 重复的循环过 程使得死锂不断累积, 造成界面阻抗增大, 循环效 率下降。

\section{1 .2 原位电子显微镜技术}

原位电子显微镜技术相比较于光学显微镜具 有更高的分辨率, 其中原位扫描电子显微镜 (SEM) 以及扫描透射显微镜(STEM)因其更大的景深获得 更富有立体感的成像，可以直接观察各种凹凸不 平的结构, 因此有助于研究者于更细致的角度研 究金属锂负极的形貌演变以及相关机理。Rong等 55 首次报道了原位电化学扫描电子显微镜(EC-SEM) 方法在锂金属电池研究中的应用, 并系统地研究 了液体电解质中的金属锂的沉积/溶解行为, 如图 11a所示。可见，得益于原位EC-SEM手段，可以更 

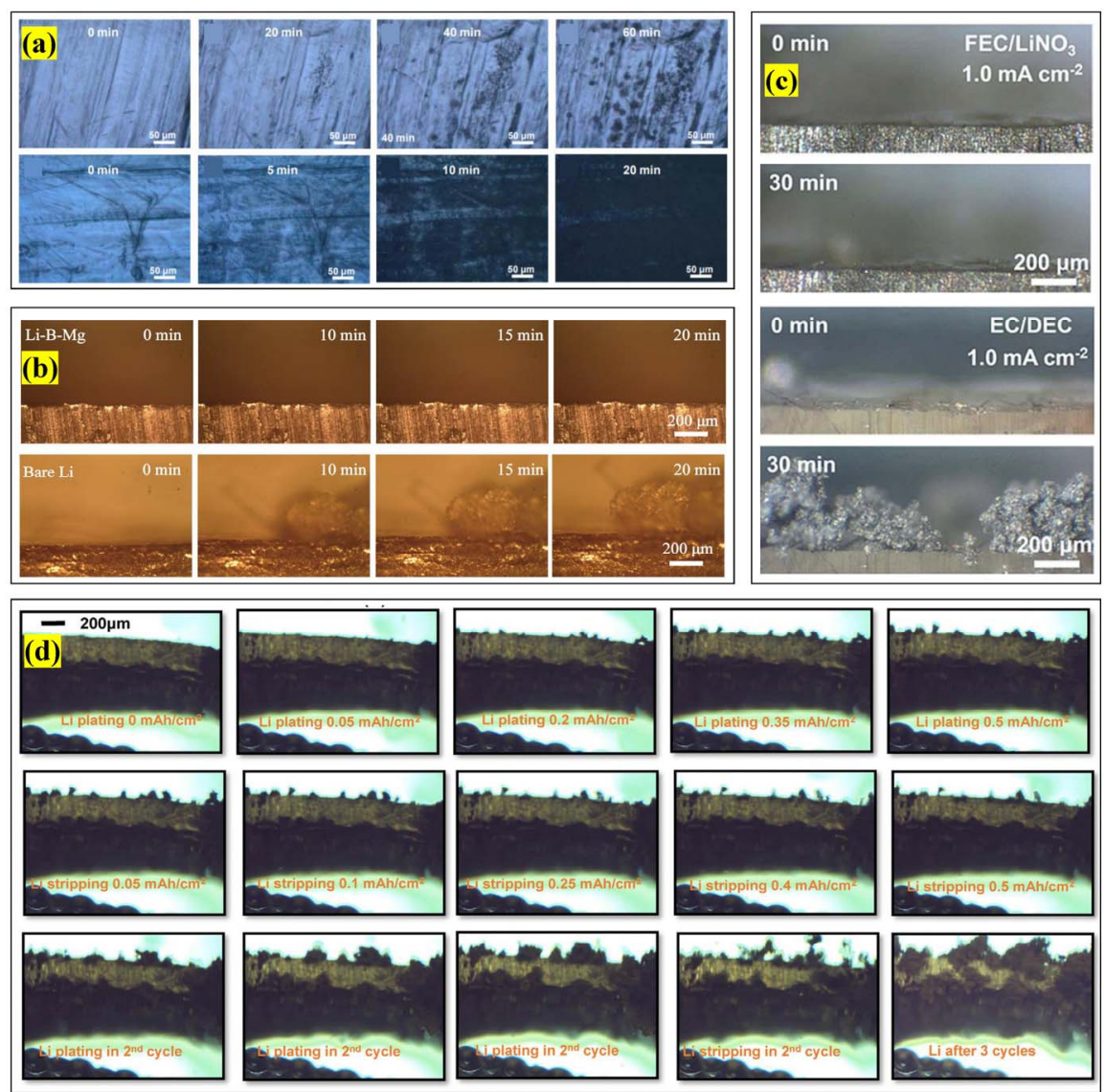

图 10 (a)金属锂电极在低和高电流密度下循环的原位光学显微镜图像 ${ }^{52} ;$ (b) Mg 掺杂 Li-LiB 合金原位光学显微镜图像 ${ }^{53}$;

(c) $\mathrm{FEC} / \mathrm{LiNO}_{3}$ 和 EC/DEC 电解液中锂的沉积形貌原位光学显微镜图像 ${ }^{54}$; (d) 金属锂负极界面原位光学显微镜图像 ${ }^{7}$

Fig. 10 The in situ observation of (a) electrode surface evolution under the low and high current density ${ }^{52}$,

(b) Li plating on the Li-B-Mg composite and pure $\mathrm{Li}$ foil ${ }^{53}$, (c) lithium deposition process in $\mathrm{FEC} / \mathrm{LiNO}_{3}$ and EC/DEC electrolytes ${ }^{54}$ and (d) the electrolyte-electrode interface on lithium metal anode ${ }^{7}$.

Adapted from American Association for the Advancement of Science, Wiley and Elsevier publishers.

加清晰和生动的反映出金属锂电极在含有 $1 \%(w)$ 硝酸锂添加剂的电解质里循环过程中的沉积/溶解 形貌变化, 其中锂枝晶和死锂的生长可通过颜色 来判断。研究结果表明, 枝晶体的生长速度和机理 受电解液中添加剂的影响很大, 且多组实验对比 发现醚基电解质中硝酸锂和多硫化锂的共添加可 最大程度地减少枝晶的生长, 并产生光滑的锂沉 积形貌。Golozar 等 ${ }^{56}$ 采用原位 SEM 研究了以 $\mathrm{LiFePO}_{4}$ 作为正极的全固态锂金属电池的循环性。如 图11b所示, 他们发现生长在固态电解质/负极界面 的枝晶并不是传统认识的锂枝晶, 而是因副反应 形成的碳化物晶体。同时, 从原位SEM照片中可以 清晰地观察到负极反应界面随着循环而出现了裂 痕, 从而形成的无压力区域促进了锂枝晶的生长。

Kim等 ${ }^{57}$ 将原位 SEM技术应用在全固态电池 的研究中。可见图 $11 \mathrm{c}$, 以LiPS作为固态电解质的 锂负极在充电和放电过程中均出现体积膨胀现
象。Hou等 ${ }^{58}$ 应用原位STEM技术对锂离子电池的 形成、生长和失效过程进行了原位观测, 研究表明 SEI膜具有双层混合的结构, SEI膜的失效则主要 与SEI膜表面的有机成分破坏后引起内层的无机 成分的溶解有关。实验通过金电极模拟金属锂表 面SEI膜的生长过程, 可见图11d, 在开始嵌锂时金 电极表面就开始形成一层厚度不均匀的SEI膜, 随 着嵌锂反应的持续进行, SEI膜的厚度也在不断的 增加。随着 $\mathrm{Li}-\mathrm{Au}$ 合金的形成我们能够在金电极表 面观察到明显的体积变化和局部凸起现象，随着 $\mathrm{Li}-\mathrm{Au}$ 合金的生长, 开始在金电极内部产生孔隙(绿 色圆圈标示位置), 在金电极和SEI膜中间的位置, $\mathrm{Li}-\mathrm{Au}$ 合金开始快速生长, 厚度达到 $3.45 \mu \mathrm{m}$, 并且 随着嵌 $\mathrm{Li}$ 的增多, $\mathrm{Li}-\mathrm{Au}$ 合金的厚度也在一直增加。

\subsection{3 原位原子力显微镜技术}

相对于光学显微镜和电子显微镜, 原子力显 微镜(AFM) 作为锂离子电池研究的好助手能通过 

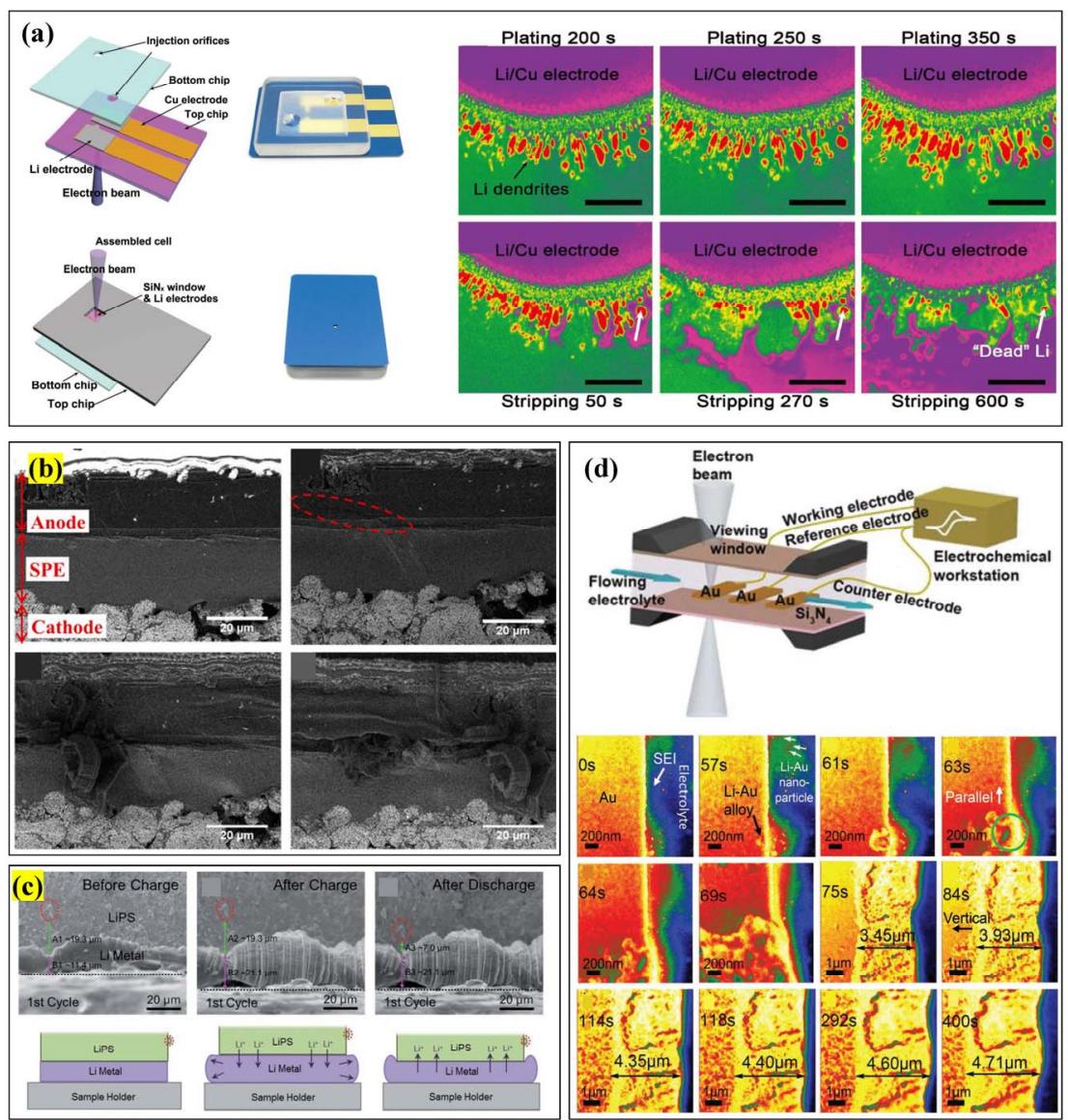

图 11 (a)金属锂电极在包含 $\mathrm{LiNO}_{3}$ 的电解夜中循环的原位 EC-SEM 表征实验装置示意图及图像 ${ }^{55}$;

(b) 固态电解质/金属锂界面的原位 SEM 图像 ${ }^{56}$; (c) NMC/LiPS/Li ASSB 首圈充放电原位 SEM 图像以及 锂金属电极体积变化示意图 ${ }^{57}$; (d)嵌锂金原位 HAADF-STEM 装置示意图及图像 ${ }^{58}$

Fig. 11 (a) Schematic diagram of the in situ SEM and the images of the electrode cycling in the ethers-based electrolyte with $\mathrm{LiNO}_{3}{ }^{55}$; (b) cross section view in situ SEM images of lithium metal ${ }^{56}$; (c) series of cross-sectional SEM images for a NMC/LiPS/Li ASSB sample and schematics for the volume change of the Li metal anode ${ }^{57}$; (d) configuration of lithium ion microcell with three electrodes for electrochemical measurements and in situ HAADF-STEM images of Au lithiation 58 .

Adapted from Wiley, American Chemical Society and Royal Society of Chemistry publishers.

其针尖原子与电极表面原子之间的相互作用来检 测电极表面的微观形貌, 并且在纳米尺度上提供 电极的物理化学信息。因此, 原位AFM技术有利 于研究金属锂电池中SEI膜和锂枝晶的物理状态, 而不仅仅是形貌特征。Liu等 ${ }^{59}$ 提出了一种简单的 一步电化学预充电法以提高锂空气电池的循环性 能, 并通过原位AFM技术直观证实了这种策略可 在惰性气氛下诱导碳纳米管、氧气扩散正极和金 属锂负极表面上形成一层薄且致密的保护膜。图 $12 \mathrm{a}$ 为在不同时间-电压条件下金属锂负极的原位 $\mathrm{AFM}$ 照片。可见, 金属锂的界面形貌在预充电前 (开路电压) 静置 $16 \mathrm{~min}$ 内没有发生明显的变化, 而 这个过程对应了电极表面惰性保护层的初步形 成。接下来, 当预充电至 $3.7 \mathrm{~V}$ 后, 可发现电极表 面钝化膜的生长较为缓慢。然而, 当超过 $3.7 \mathrm{~V}$ 时,
由于电解液的快速分解造成金属锂界面形貌发生 了明显的变化。最后, 在保持 $4.7 \mathrm{~V}$ 条件下运行 20 $\min$ 后发现保护层的结构逐渐平整且连续性提高。 Wang等 ${ }^{60}$ 采用原位 AFM技术详细研究了不同电解 液体系下对应的锂枝晶在石墨负极上的生长状 态, 如图 $12 \mathrm{~b}$ 所示。当采用 $1 \mathrm{~mol} \cdot \mathrm{L}^{-1} \mathrm{LiPF}_{6} / \mathrm{EC} / \mathrm{DMC}$ 电解液时, $\mathrm{AFM}$ 等高线图显示经过 $4 \mathrm{~min}$ 放电后的 石墨电极表面发生了锂析出现象, 且进行 $8 \mathrm{~min}$ 后 的电极表面沉积出一层颗粒状的金属锂。这说明 在 $\mathrm{EC} / \mathrm{DMC}$ 体系中的石墨负极存在严重的析锂现 象, 从而安全性较低。作为对比, 原位 $\mathrm{AFM}$ 等高线 图显示石墨电极嵌锂时的表面形貌在FEC/DMC 电解液体系中基本不发生变化。日本Kitta团队 ${ }^{61}$ 采 用原位AFM技术研究了金属锂表面的锂沉积机理 以及SEI膜的力学性能, 可见图 $12 \mathrm{c}$ 。在初始锂沉积 

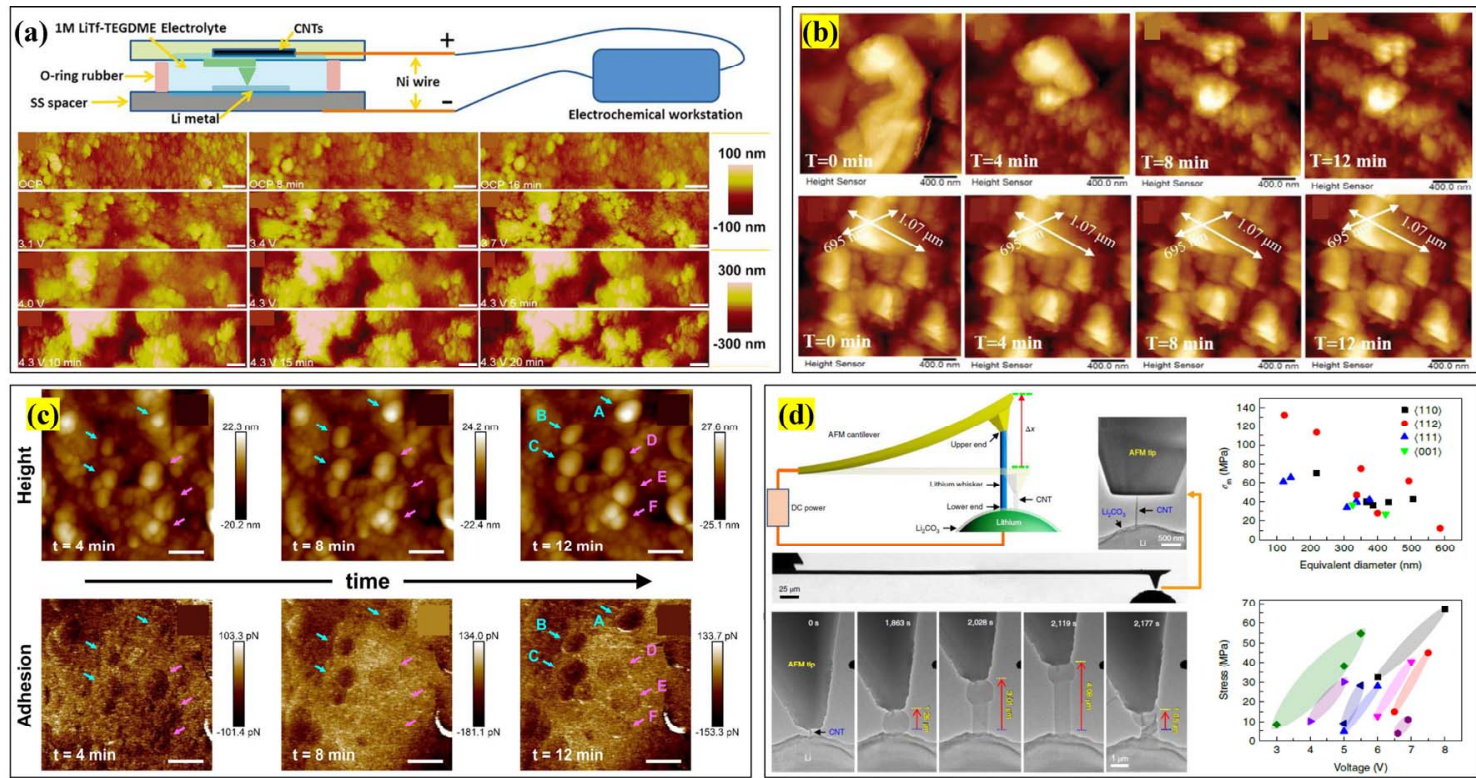

图 12 (a) 原位 AFM 设备示意图和不同状态时金属锂表面保护层的 AFM 照片 ${ }^{59}$; (b) 在 EC/DMC 和 FEC/DMC 体系中 锂沉积时电极表面原位 AFM 照片 ${ }^{60}$; (c)锂沉积过程的原位 AFM 照片与表面粘附力变化 ${ }^{61}$;

(d) 原位 AFM-ETEM 技术在研究锂枝晶生长中的应用 62

Fig. 12 (a) Schematic diagram of the in situ AFM and the corresponding images of the Li electrode surface under different conditions ${ }^{59}$; (b) in situ AFM images of electrode surface during the Li deposition at EC/DMC and FEC/DMC systems ${ }^{60}$; (c) in situ AFM and adhesion mapping images of the electrode during the Li plating ${ }^{61}$; (d) application of in situ AFM-ETEM technology in the study of lithium dendrite growth ${ }^{62}$.

Adapted from Wiley, American Chemical Society and Springer Nature publishers.

过程中，原位AFM的等高线照片显示在电极表面 形成了两组锂突触结构(红色和蓝色箭头), 且蓝色 锂突触组的尺寸随沉积的进行而逐渐变大, 相反 红色组没有发生明显的变化。同时, 由AFM衍生 出的粘附力测试表明蓝色箭头标识的锂突触具有 比红色组突触更低的粘附力, 说明金属锂表面的 弱粘附力与锂枝晶的生长具有紧密的联系。

另一方面, 原位AFM也可以与其他成像技术 连用来提高金属锂研究的准确性和信息量。Zhang 等 ${ }^{62}$ 采用先进的原位原子力显微镜-环境透射电子 显微镜(AFM-ETEM)技术, 实时、直观地记录了锂 枝晶生长的微观机制, 且精准测定了枝晶的力学 性能, 同时提出了固态电池中抑制锂枝晶生长的 可行性方案。图12d为原位AFM-ETEM设备的示意 图, 其中包含了金属锂负极、碳酸锂固态电解质以 及硅针尖对电极。可见, 在锂沉积过程中, AFM单 元一方面可作为电极以触发理枝晶的生长, 而另 一方面其对生长过程中的锂枝晶产生了一定的约 束力, 从而实现对锂枝晶物理性质的实时观察。他 们通过这项技术首次发现在室温条件下亚微米晶 须在AFM尖端的外加电压(过电位)下生长可产生 高达 $130 \mathrm{MPa}$ 的生长应力, 对应的屈服强度高达
$244 \mathrm{MPa}$, 明显高于先前报导的块体和微米尺寸锂 的应力。

\section{1 .4 其他原位表征技术}

除了电子显微镜和光学显微镜技术外, $X$ 射线 成像技术在近几年得到了快速的发展, 它具有对 样品无损伤、精度高、快捷、无污染等优点, 同时 可以得到相关晶体的准确信息以便进行深入的分 析。Sun等 ${ }^{63}$ 采用无损原位X射线成像技术分别研 究了锂铝合金负极、PVDF-锂负极、阳极氧化铝 (AAO)膜-锂负极体系的界面变化机理, 如图13a所 示。在该种高新技术的帮助下能够清晰地观察到 锂铝合金负极体系中在锂沉积初期电极表面形成 了球状锂成核位点, 且反应界面形貌随着循环的 进行而不断更新, 最终形成了具有高比表面积的 锂沉积物(HSAL)。同时, 他们采用更高分辨率的 X射线成像技术进一步观察了 HSAL的生长细节。 可见, 电极循环一周后的X射线成像3D形貌图表 明三种电池体系在循环效率大于 $99 \%$ 的情况下仍 会出现HSAL现象。通过该原位、无损的先进表征 手段技术可以将电池整体的电化学性能研究与电 极材料的形貌表征结合在一起研究以得到更完整 的信息, 这将有利于进一步认识电化学沉积过程 

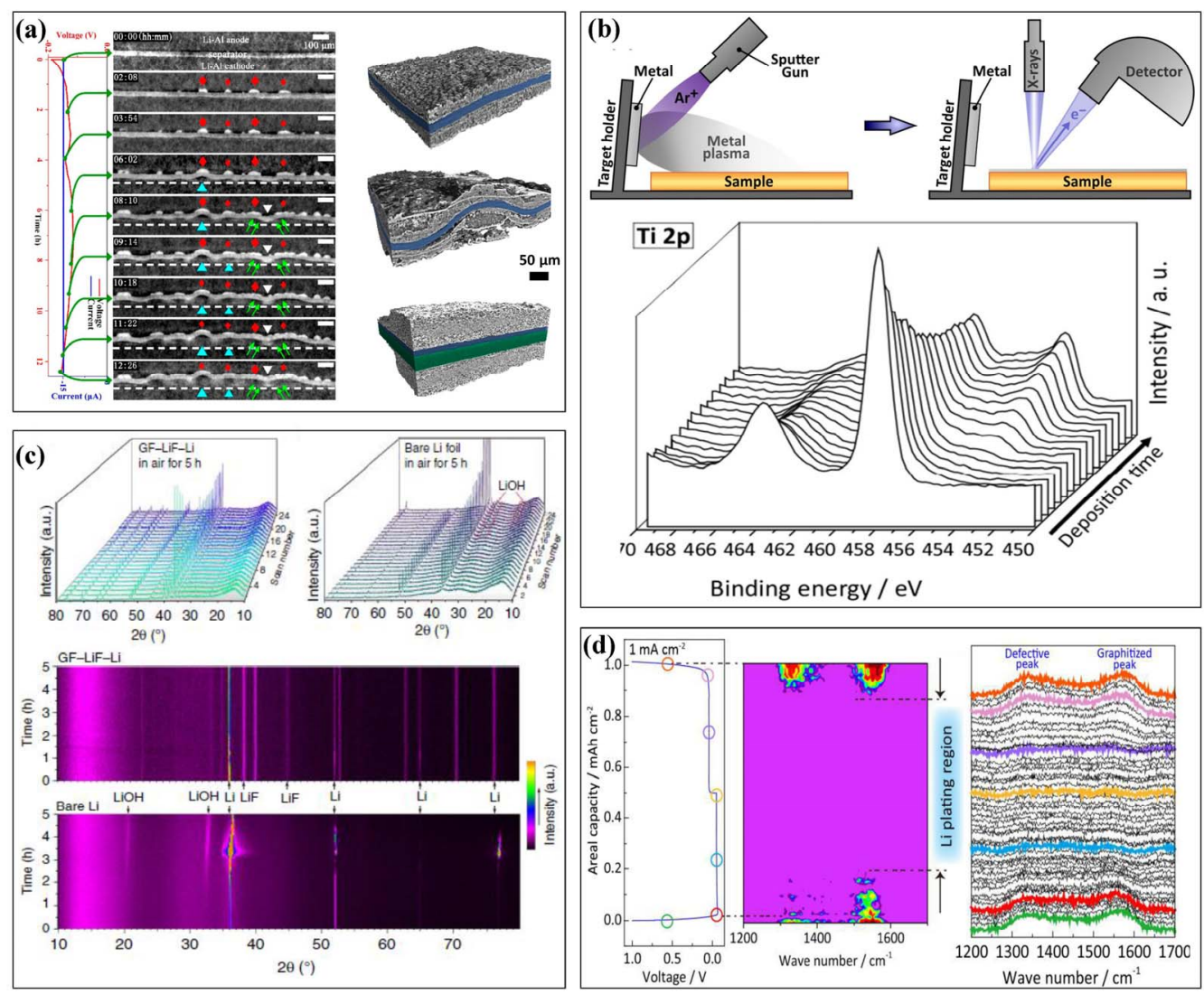

图 13 (a)首次放电时锂铝合金对称电池的原位 $X$ 射线成像照片以及三种体系的 3D 渲染图 ${ }^{63}$;

(b)原位 XPS 用于研究固态电解质/金属锂界面的示意图和 LLTO 在锂沉积过程中的 Ti $2 p$ XPS 谱的变化 ${ }^{64}$;

(c) GF-LiF-Li 和 bare Li 在大气环境中的原位 XRD 测试 ${ }^{65}$; (d)锂沉积/溶解过程中的原位 Raman 谱图 66

Fig. 13 (a) In operando X-ray tomography characterization of the symmetric cell using Li-Al alloy electrode and corresponding 3D rendering of three samples ${ }^{63}$; (b) schematic diagram of the research soild electrolyte/Li metal interface via in situ XPS technique, and the change of Ti $2 p$ XPS of LLTO during the Li deposition ${ }^{64}$; (c) in situ XRD images of GF-LiF-Li and bare Li standing in ambient atmosphere ${ }^{65}$; (d) in situ Raman spectrum of the L-DG host during the $\mathrm{Li}$ plaing and stripping ${ }^{66}$.

Adapted from American Chemical Society, Elsevier and Springer Nature publishers.

中锂枝晶微形貌的形成机制以及演化机制。Janek 等 ${ }^{64}$ 采用原位X射线光电子能谱(XPS)技术探究了 固态电池中固态电解质/金属锂界面的形成与变 化。设备原理示意图如图13b所示, 可见实验的核 心在于通过XPS中的氩离子枪对辅助金属锂进行 溅射而触发锂沉积行为, 同时对固态电解质进行 实时的XPS检测以研究沉积界面发生的化学反 应。原位XPS测试清晰地表明LLTO (锂针钛氧)固 态电解质中的 Ti $2 p$ 信号在锂沉积过程中发生了明 显的变化。原始界面的XPS表明只存在Ti的一种氧 化态 $\left(\mathrm{Ti}^{4+}\right)$, 而随着锂沉积的进行, 接连在低结合 能处出现了 $\mathrm{Ti}^{3+} 、 \mathrm{Ti}^{2+}$ 和金属 $\mathrm{Ti}$, 表明LLTO的锂嵌 入过程。

Goodenough 课题组 ${ }^{65}$ 通过原位 X 射线衍射 (XRD)技术对不同种类金属锂电极在环境气氛中
的物相变化进行了检测, 如图13c所示。在相对湿 度为 $10 \%$ 条件下, 原位 XRD显示锂片的衍射峰皆 发生了一定程度的位移, 并且强度相对提升, 表明 锂片在大气环境中与氧气和水发生了剧烈的反 应, 导致锂片被刻蚀。作为对比, GF (氟化石墨)$\mathrm{LiF}-\mathrm{Li}$ 电极的原位XRD谱图随着时间进行而没有 发生明显的变化, 说明GF-LiF层有效地抑制了水 与锂金属之间的反应, 从而可以作为金属锂表面 的一种保护涂层。拉曼(Raman)光谱技术可用来检 测材料的分子构型, 从而广泛的应用在材料领域 的研究中。Qiao等 ${ }^{66}$ 在贫液电解液体系对锂沉积影 响的工作中采用了一种原位Raman技术来检测碳 缺陷对锂成核的作用, 如图13d所示。在这里, 虽 然Raman技术无法探测金属的结构(金属锂), 但可 以通过对比锂沉积前后的碳基质 Raman谱图来侧 
面反映锂的成核行为。他们发现在锂沉积过程中, 位于原位 Raman谱图中 $1350 \mathrm{~cm}^{-1}$ 处的碳缺陷峰 首先消失, 而位于 $1580 \mathrm{~cm}^{-1}$ 的石墨化结构峰在进 一步沉积后也逐渐消失。这说明锂的成核沉积过 程在初期更倾向发生在碳基质表面的缺陷处, 因 而这些区域具有更高的亲锂性。而在随后的沉积 中, 锂逐渐覆盖碳基质表面, 从而石墨化结构被金 属锂遮挡。

\section{2 冷冻电镜技术}

冷冻电镜技术(cryo-EM)近年来取得了快速的 发展, 推动了各领域科研工作的进步与理论认识 的深入。这种技术是将电镜与超低温冷冻制样及 传输策略相结合, 以实现直接观察液体、半液体和 对电子束敏感的样品, 如生物材料、高分子材料 等。冷冻电镜装置一般是在电镜主体仪器, 例如透 射电镜、扫面电子显微镜和电子显微镜等上加装 样品冷冻模块 67 。冷冻透射电镜的装置示意图可见 图14a。该技术最初主要应用于结构生物学研究, 而近几年逐渐应用于材料化学领域, 尤其是涉及 金属锂电池中锂枝晶的研究。众所周知, 金属锂对 水分、氧气、氮气、二氧化碳和光束等都十分敏感,
因而很难采用传统的高能电子显微镜来表征其界 面形貌的变化以及纳米尺寸锂枝晶的结构 68 。受结 构生物学的启发, 冷冻电子显微镜提供了一种可 以使电池保持自然状态并以纳米和原子级尺度对 电池材料进行成像的表征方法。低温保护不仅有 助于最大程度地减少空气和光束的损害, 而且还 保留了样品的固有结构。这使我们能够在纳米/原 子级分辨成像轻质化合物的结构与形貌, 而这对 锂枝晶的生长以及SEI膜的研究至关重要。

$\mathrm{Li}$ 等 69 利用冷冻电镜观察到碳酸盐基电解质 中完整的树枝状锂枝晶晶体。如图14b所示, 枝晶 结构十分清晰, 且能谱测试结果显示金属锂在冷 冻传输过程中得到了很好的保存。作为对比, 室温 下高分辨透射电镜中观测的锂枝晶呈现更粗龯的 表面和更深的对比度, 说明样品表面在环境气氛 中形成了多晶氢氧化锂(即使暴露的时间很短)。同 时, 锂枝晶样品被高分辨电镜的强电子束穿透而 形成了多个孔洞形貌(如图中白色亮点所示)。可 见, 冷冻电镜技术对锂枝晶的观察更加准确。此 外, 他们通过这项技术发现锂枝晶在不同方向上 的横截面呈现不同的多面体形状，分别有三角形，

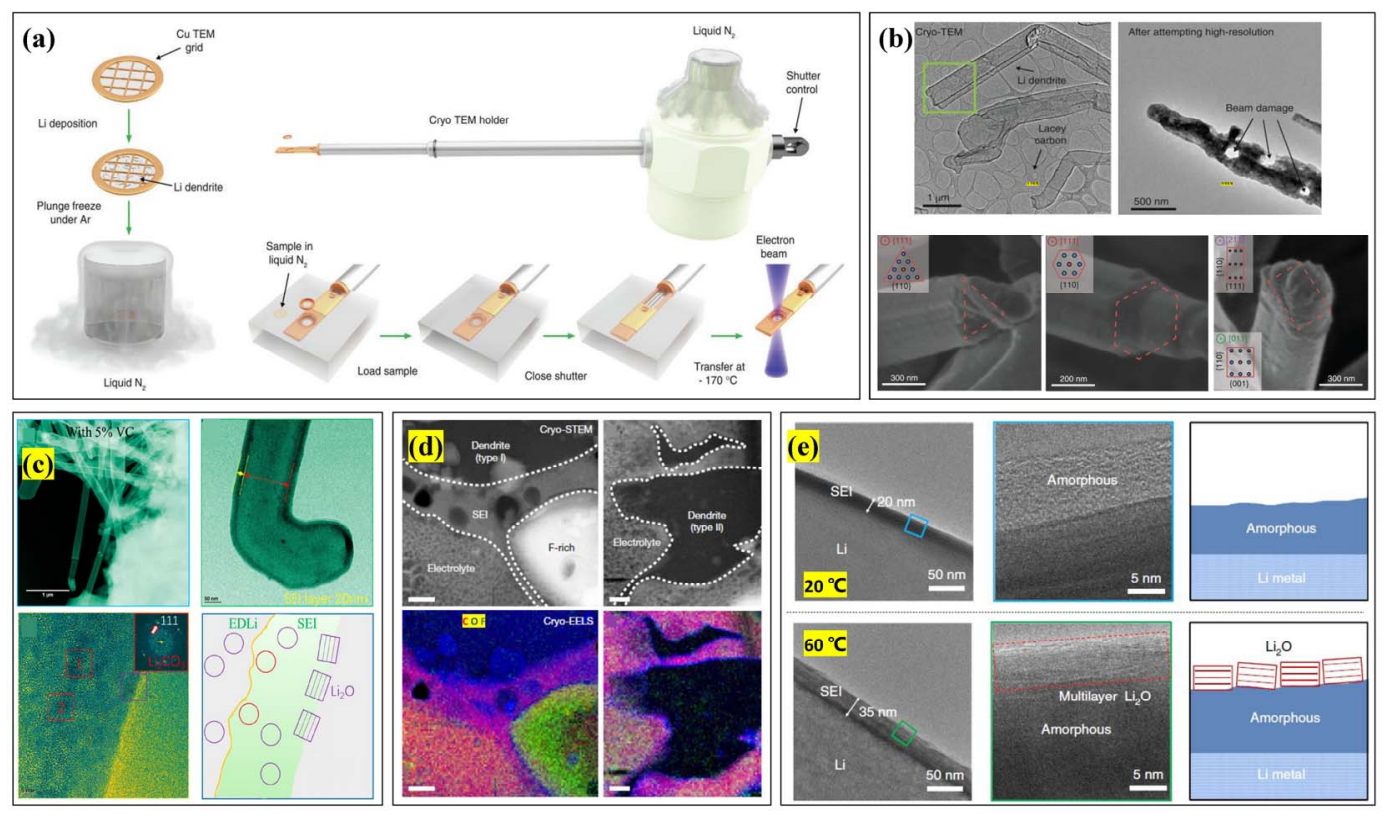

图 14 (a)冷冻电镜实验操作示意图 ${ }^{68}$; (b)锂枝晶 cryo-TEM 与普通 TEM 图像对比图和锂枝晶不同截面 cryo-SEM 图 ${ }^{69}$; (c)含 VC 的电解质中锂枝晶低放大倍数、明场和原子级分辨率 cryo-TEM 图像 ${ }^{70}$; (d) 类型 I 和 II 锂枝晶的 cryo-STEM 和

EELS 图像 ${ }^{71}$; (e)使用 $1 \mathrm{~mol} \cdot \mathrm{L}^{-1}$ LiTFSI 盐在 20 和 $60^{\circ} \mathrm{C}$ 下所形成 SEI 界面的 cryo-EM 图像和纳米结构示意图 72

Fig. 14 (a) Preserving and stabilizing Li metal by cryo-EM 68; (b) cryo-TEM and ordinary TEM images of Li dendrite, and the cryo-SEM images of different cross section of Li dendrite ${ }^{69}$; (c) low magnification, bright-field and atomic-resolution of

EDLi and SEI formed in VC-containing electrolyte ${ }^{66}$; (d) cryo-STEM and EELS images of type I and II dendrite ${ }^{71}$;

(e) cryo-EM images and nano-structure diagrams of dendrites growth at 20 and $60{ }^{\circ} \mathrm{C}$ with the $1 \mathrm{~mol}^{\circ} \mathrm{L}^{-1} \mathrm{LiTFSI}^{\mathrm{salt}}{ }^{72}$.

Adapted from Elsevier, American Association for the Advancement of Science, American Chemical Society and Springer Nature publishers. 
六边形和长方形截面, 而普通扫描电镜无法观察 到这一现象。除了基础形貌表征外, 高分辨率的透 射电镜(TEM)、能量色散X射线光谱(EDS) 以及电 子能量损失光谱(EELS)等测试手段在冷冻电镜技 术的帮助下可更细致和全面地描述锂的沉积形貌 以及SEI膜的结构与化学成分分布。 $\mathrm{Xu}$ 等 70 通过冷 冻电镜技术揭示了碳酸亚乙烯酯 $(\mathrm{VC})$ 作为锂金属 电池电解质添加剂的作用机理。他们发现在含 $\mathrm{VC}$ 的电解质中沉积的金属锂呈现出带状形貌 (图 $14 \mathrm{c}$ ), 且明场像表明沉积物仅被轻微氧化。同时, 高分辨TEM图像表明存在于锂沉积物表面的 SEI 膜由 $\mathrm{Li}_{2} \mathrm{O}$ 和 $\mathrm{Li}_{2} \mathrm{CO}_{3}$ 组成, 且呈现出清晰的纳米级 马赛克状结构。

固液界面的性质与变化在化学、物理和生物 研究中十分重要, 但由于缺乏与固液成分兼容的 高分辨率表征方法导致人们对固液界面的某些行 为和概念还认识不清。Zachman等 ${ }^{71}$ 采用冷冻扫描 透射电子显微镜(cryo-STEM)方法研究了固液界 面处锂枝晶的结构和组成。通过对液体电解质进 行玻璃化处理可以将电池固液界面的结构保留为 原始状态, 从而可以更加清晰与准确地观察两相 界面的演变状态, 如图14d所示。研究发现锂金属 负极上共存着两种具有不同的结构和组成的枝晶 类型, 一种锂枝晶具有扩展SEI界面层, 而另一种 则没有。同时, 冷冻电子能量损失光谱测试(cryoEELS)对比显示扩展的I型SEI具有更高的氧和锂 浓度且基本不含氟元素。长期以来, 锂离子电池的 工作温度区间一直限于接近室温的狭窄温度范 围。而在更高的温度下, 由于两相界面副反应增强 导致电池的循环性能迅速衰减。特别是采用高反 应活性的金属锂作为负极时, 所形成的反应界面 在高温条件下演变的更加复杂与剧烈, 且对电池 电化学性能的影响程度更大。Cui等 ${ }^{72}$ 利用冷冻电 镜研究了高温下运行的金属锂电池的电化学性能 以及界面结构变化, 如图 $14 \mathrm{e}$ 所示。可见, 在 $20^{\circ} \mathrm{C}$ 和 $60{ }^{\circ} \mathrm{C}$ 条件下循环后的金属锂电极表面SEI膜结 构截然不同。发现在较高的温度下形成的SEI膜具 有更高的厚度, 且在无定形层SEI膜的表面形成了 一层新的多级层, 而该结构具有更高的机械稳定 性, 可抑制两相界面处连续发生的副反应并确保 良好的循环稳定性和低电化学阻抗。

\section{3 中子深度分析}

中子深度分析(NDP)是一种非破坏性的中子 分析技术, 不仅对锂具有良好的灵敏度, 而且对微 米级的深度具有优异的空间分辨率。它既可以作 为独立技术使用, 也可以与其他多种分析方法配
合使用 ${ }^{73}$ 。这种分析技术目前已经应用于金属锂电 池的研究中, 并且是实现深入探索锂枝晶生长模 式的一种有效研究方法。

马里兰大学Wang团队 ${ }^{74}$ 通过原位NDP技术研 究了三种具有代表性的固态电解质(LiPON, LLZO (锂锞锆氧) 和非晶 $\mathrm{Li}_{3} \mathrm{PS}_{4}$ ) 的 $\mathrm{Li}$ 浓度分布在锂沉积 过程中的动态演变, 并以此来解释了锂枝晶的形 成起源(图15a)。实验发现在锂沉积过程中, LiPON$25^{\circ} \mathrm{C}$ 的体相区域中的锂含量保持恒定, 从而固态 电解质没有枝晶生成。然而, LLZO和 $\mathrm{Li}_{3} \mathrm{PS}_{4}$ 则在 不同运行温度下均有枝晶生成, 且温度越高枝晶 生长的越快。同时, 他们揭示了高电子电导率可能 是固态电解质内枝晶发展的原因, 并指出降低固 态电解质电子电导率可能是全固态电池安全性方 面更亟待解决的问题。原位NDP技术可以通过对 锂浓度分布的动态演变检测获得更精确全面的信 息, 这对锂枝晶的生长机理研究至关重要。Li课题 组 ${ }^{75}$ 将原位中子深度分析与同位素方法结合, 定量 地解析了金属锂电池沉积/溶解过程的锂元素在空 间分布的不均匀性, 对安全高性能锂金属电极的 开发和应用具有重要的指导意义。图 $15 \mathrm{~b}$ 为在 1.0 $\mathrm{mA} \cdot \mathrm{cm}^{-2}$ 电流密度下的四个循环过程中锂密度与 垂直于 $\mathrm{Cu}$ 集流体深度的函数, 可见电极反应界面 在重复循环中形成了密度更低且更厚的锂沉积 物, 且这些轮廓的低密度尾部特征表明高度多孔 的锂沉积物逐渐延伸到了电解质内部。

NDP技术可以测量锂在某一方向上的含量以 及分布情况。它作为目前唯一直接测量锂元素的 表征技术, 具有很高的灵敏度和精确性。 $\mathrm{Hu}$ 等 ${ }^{76}$ 采 用原位NDP技术研究了固态电池中 $3 \mathrm{D}$ 碳纳米管 (CNT) 电极/石榴石 (Garnet) 固态电解质的界面变 化和锂沉积/溶解行为。图 $15 \mathrm{c}$ 显示了 CNT电极结构 在充放电过程中的内部锂含量变化, 其中 2150 到 $2500 \mathrm{keV}$ 之间的能量对应于碳纳米管薄膜的表 面。首先, 随着循环次数的提高, CNT区域的 ${ }^{3} \mathrm{H}$ 数 量随之增加, 表明锂在循环时逐渐在 CNT网络中 积聚。同时, 原位NDP的等高线图清晰表明了大量 的锂在循环后堆积在 $\mathrm{CNT}$ 的表面而没有参与反 应。另外, 在一次循环中, 锂沉积截止时对应的 CNT 近表面(靠近Garnet) ${ }^{3} \mathrm{H}$ 数量最高, 而溶解截止时对 应的 ${ }^{3} \mathrm{H}$ 数量最低, 表明锂较为可逆的沉积/溶解反 应。这表明在CNT电极的结构中, 靠近Garnet的 CNT层具有较高的可逆性。当沉积容量过大时, 生 长在非CNT近表面的金属锂由于与Carnet较差的 接触性而失去了反应活性, 从而逐渐在 CNT电极 中累积。 $\mathrm{Li}$ 等 77 制备了一种 $\mathrm{Li} \mid \mathrm{Li}_{6.4} \mathrm{La}_{3} \mathrm{Zr}_{1.4} \mathrm{Ta}_{0.6} \mathrm{O}_{12}$ 

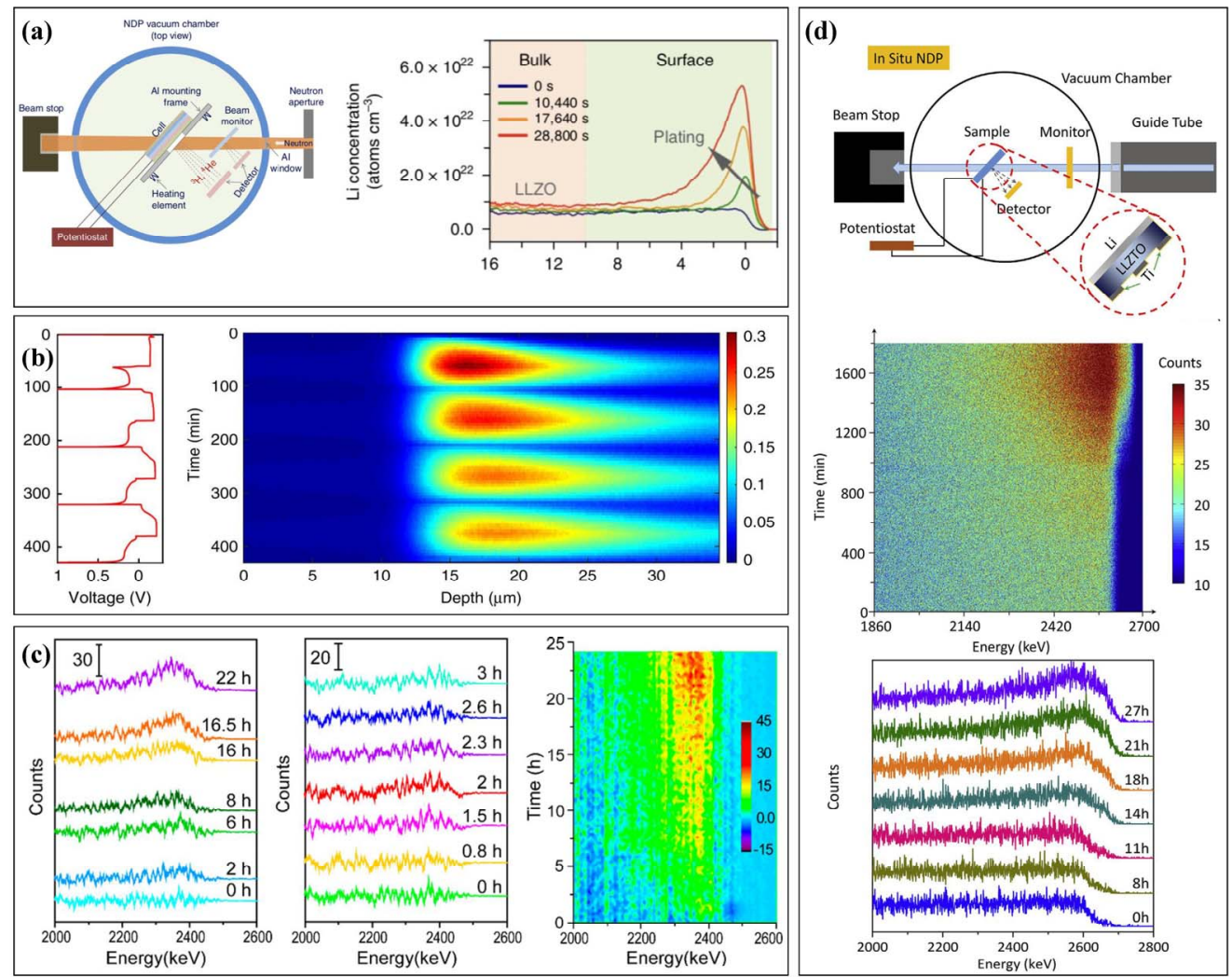

图 15 (a)原位 NDP 原理示意图与 Li/LLZO/Cu 电池的时间分辨的锂浓度曲线 ${ }^{74}$; (b) 原位 NDP 测量金属锂循环时的 相对锂密度与深度关系图 ${ }^{75}$; (c) Li/garnet/CNT 电池 NDP 图像 ${ }^{76}$; (d) 原位 NDP 示意图及 NDP 图像 ${ }^{77}$

Fig. 15 (a) Schematic of the experimental set-up for operando NDP and time-resolved lithium concentration profiles for $\mathrm{Li} / \mathrm{LLZO} / \mathrm{Cu}$ cell ${ }^{74}$; (b) operando NDP measurements of $\mathrm{Li}$ metal electrode during the cycling ${ }^{75}$;

(c) in situ NDP measurement of a Li/garnet/CNT asymmetric cell ${ }^{76}$; (d) experimental setup for the in situ NDP measurements and in situ NDP spectra ${ }^{77}$.

Adapted from Springer Nature, American Chemical Society and Elsevier publishers.

(LLZTO) $\mid \mathrm{Ti}$ 全固态锂电池, 并通过原位NDP技术 检测了锂在电极/电解质界面的沉积取向。图15d为 多孔Ti电极在锂沉积过程中的原位NDP谱图, 且 不同的能量范围对应不同的材料深度。可见，图中 ${ }^{3} \mathrm{H}$ 的数量随着沉积的进行而逐渐提高, 且 $2560-$ $2630 \mathrm{keV}$ 区间的变化最为显著, 同时明显可以看 到曲线的峰值在 $2595 \mathrm{keV}$ 左右略微变化, 表明锂 沉积过程重要发生在 $\mathrm{Ti}$ 电极的孔道里, 而不是电 极表面。此外, 随着时间的进一步增加, 位于 2595 $\mathrm{keV}$ 的峰的垂直边缘向外延伸, 且 ${ }^{3} \mathrm{H}$ 数量迅速增 加, 表明锂能够在 Ti电极的孔道空间中沉积并持 续生长。通过原位 NDP探测到的这种锂沉积行为 表明, 3D Ti电极可最大限度抑制固态电解质的衰 败和锂枝晶的形成, 从而提高电池的电化学性能。

\section{4 固态核磁共振技术}

固态核磁共振 (ssNMR) 技术是以固态样品为 研究对象的一种无损的分析技术, 可以反应出不 溶固体或者溶解后结构发生变化的固体的结构信
息, 广泛应用于在物理、化学、材料和矿物等方面 的研究中。相比于传统的X射线衍射技术、振动光 谱(红外光谱和拉曼光谱)等结构表征技术, ssNMR 技术主要研究固体中某种特定的短程有序局部环 境, 通过ssNMR谱图中的化学位移变化来考察原 子核与原子核之间的相互作用及各原子的局部微 环境, 可探索详尽的原子周围局部结构信息, 因此 可以研究长程无序的非晶材料或结晶度低的固体 物质。ssNMR技术因其独特的优势被广泛应用于 金属锂电池研究中, 包括固态电解质结构信息研 究和电解液固体产物监测等, 对于深入理解电池 材料的结构信息十分关键。

Zheng等 ${ }^{78}$ 用立方相 $\mathrm{Li}_{7} \mathrm{La}_{3} \mathrm{Zr}_{2} \mathrm{O}_{12}$ (LLZO)石榴 石和聚氧化乙烯 $(\mathrm{PEO})$ 制备了复合固态电解质 (LLZOPEO), 并将其应用于对称锂电池中, 通过结 合选择性同位素标记和高分辨率固态核磁技术 (图16a), 实现了监测电池循环过程中 ${ }^{6} \mathrm{Li}$ 金属电极 与复合电解质中 ${ }^{7} \mathrm{Li}$ 的置换, 跟踪LLZOPEO复合电 
(a)
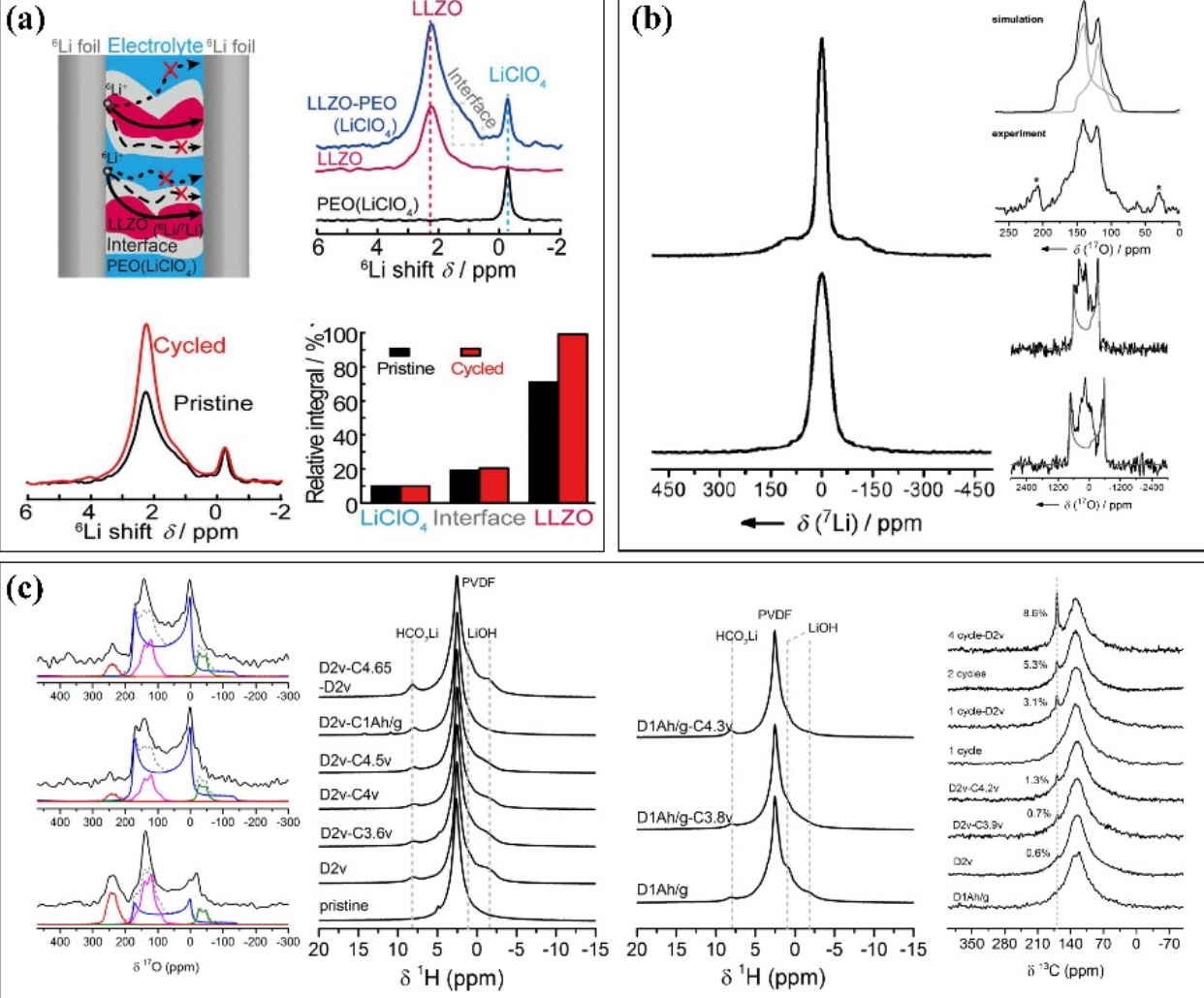

图 16 (a) 对称 ${ }^{6} \mathrm{Li}$ foil/复合电解质/ $/ \mathrm{Li}$ foil 电池电解质中 $\mathrm{Li}^{+}$传输路径示意图, LLZO-PEO $\left(\mathrm{LiClO}_{4}\right)$ 复合电解质循环前后 ${ }^{6} \mathrm{Li} \mathrm{NMR}$ 谱对比及循环前后 ${ }^{6} \mathbf{L i}$ 含量的定量分析 ${ }^{78}$; (b) 电池 A 和电池 B 正极的单脉冲静态 ${ }^{7} \mathbf{L i}$ 和 ${ }^{17} \mathrm{O}$ 核磁共振谱 ${ }^{79}$; (c)正极在不同充电状态下的 ${ }^{17} \mathrm{O} 、{ }^{1} \mathrm{H}$ 以及 ${ }^{13} \mathrm{C} \mathrm{Hahn}$ 回波谱 ${ }^{80}$

Fig. 16 (a) Illustration of the symmetric ${ }^{6} \mathrm{Li}$ foil/composite electrolyte $/{ }^{6} \mathrm{Li}$ foil battery , possible $\mathrm{Li}^{+}$transport pathways, comparison of the ${ }^{6} \mathrm{Li}$ NMR spectra before (pristine) and after (cycled) cycling, and quantitative analysis of ${ }^{6} \mathrm{Li}$ amount ${ }^{78}$;

(b) one-pulse static ${ }^{7} \mathrm{Li}$ and ${ }^{17} \mathrm{O}$ NMR spectra of the discharged cathode from cell $\mathrm{A}$ and cell $\mathrm{B}{ }^{79}$; (c) ${ }^{17} \mathrm{O}$, ${ }^{1} \mathrm{H}$ and

${ }^{13} \mathrm{C}$ Hahn echo spectra of cathodes at different states of charge ${ }^{80}$.

Adapted from Wiley and American Chemical Society publishers.

解质中锂离子的路径。ssNMR技术还被广泛应用 在金属锂-空气电池的研究中, 监测循环过程中形 成的电化学产物的演变, 以进一步理解影响电池 容量衰减的因素。Leskes等 ${ }^{79}$ 首次使用 ${ }^{7} \mathrm{Li}$ 和 ${ }^{17} \mathrm{O}$ 核 磁共振波谱的组合, 研究了有机碳酸盐基电解液 体系中的锂-空气电池在放电过程中电解液的分 解产物。如图16b所示, ssNMR测试结果结合密度 泛函理论(DFT)计算结果共同证明了在标准有机 碳酸盐基电解液体系中的锂-空气电池在放电过 程中电解液分解形成的主要产物为碳酸锂和过氧 化锂。Leskes课题组 ${ }^{80}$ 在后续工作中进一步研究了 1,2-二甲氧基乙烷(DME)基电解质体系中锂-空气 电池的电化学产物, 通过对 ${ }^{17} \mathrm{O} 、{ }^{6} \mathrm{Li} 、{ }^{7} \mathrm{Li} 、{ }^{1} \mathrm{H}$ 和 ${ }^{13} \mathrm{C}$ ssNMR的多核研究, 鉴定并量化了在碳电极的孔 和表面形成的多种不溶性产物。图 $16 \mathrm{c}$ 显示的 ${ }^{17} \mathrm{O}$ ssNMR结果表明首次放电主要产物为过氧化锂, 而在充电过程中的 ${ }^{13} \mathrm{C} \mathrm{ssNMR}$ 结果显示正极表面
形成了碳酸盐, 并在第一个循环的高充电电压下 消失, 但在随后的循环中逐渐积累。SSNMR技术在 直接检测电池内产物形成和分解方面具有独特的 优势, 是评估新型电解质、催化剂和电极材料的重 要检测手段。

上述先进表征手段虽然为深入认识金属锂的 失效机制提供了重要信息，但每种技术都有其自 身局限性。原位表征技术对原位电池和工作环境 的要求苛刻, 而冷冻电镜、中子深度分析和固态核 磁共振技术存在制样复杂，实验资源稀缺等问题。 此外, 目前金属锂负极界面的演变和电极内部压 力分析方面的表征非常重要但极具挑战, 急需开 发针对金属锂负极界面和内部压力分析的新表征 技术。

\section{4 总结与展望}

得益于金属锂的高理论容量与低氧化还原电 
位, 金属锂负极在未来锂电池的发展中极具潜力。 然而, 其锂枝晶生长和不稳定的界面结构所引发 的性能衰减与安全隐患问题也非常突出。本文从 SEI膜、锂枝晶和死锂层三个方面分析了金属锂负 极的失效机制, 总结了SEI膜的形成机理(Peled型、 马赛克型、双层型、多层型和库伦反应型)及金属 锂表面SEI膜的特征。总结发现锂枝晶的形成机制 包括成核/扩散模型、异相成核模型、空间电荷模 型和SEI膜诱发成核模型。在循环过程中, 由于金 属锂的不均匀溶解使得锂枝晶脱落所形成的 “死 锂” 层, 是其性能衰降的另外一个重要因素。

解决金属锂负极的失效问题是实现金属锂电 池商业化的必经之路, 如何发挥金属锂电极的优 势并限制其弊端是锂电池领域科研人员必须要去 面对的挑战。关于锂枝晶或电极界面性质的基础 理论研究始终在进行。总结相关文献可以看出, 金 属锂问题的机理研究以 2015 年为节点分为两个阶 段。2015年之前主要集中在锂枝晶生长与形态的 研究和相关理论计算。然而受到实验条件和表征 技术的限制, 关于锂枝晶生长的理论一直存在争 论。2015年之后, 随着一系列先进表征技术的发展 与应用, 研究人员得以从多角度对锂枝晶的生长、 死锂的形成以及反应界面结构进行了深入和精准 的解析。近年来, 研究人员在解决金属锂失效问题 方面提出了多种策略, 包括电解液改性、人工SEI 膜构建、固态电解质引入、宿主骨架复合等, 取得 了很多瞩目的成果, 提升了金属锂负极的性能。相 信通过科研人员的不断努力和先进表征技术的进 一步发展和运用, 金属锂负极的失效问题终将得 以解决。

\section{References}

(1) Armand, M.; Tarascon, J. Nature 2008, 451, 652. doi: $10.1038 / 451652 \mathrm{a}$

(2) Guo, Y.; Li, H.; Zhai, T. Adv. Mater. 2017, 29, 1700007. doi: 10.1002/adma.201700007

(3) Grande, L.; Paillard, E.; Hassoun, J.; Park, J. U.; Scrosati, B. Adv. Mater. 2014, 27, 784. doi: 10.1002/adma.201403064

(4) Kolosnitsyn, V. S.; Karaseva, E. V. Russ. J. Electrochem. 2008, 44, 506. doi: 10.1134/s1023193508050029

(5) Xu, W.; Wang, J.; Ding, F.; Chen, X.; Nasybulin, E.; Zhang, Y.; Zhang, J. Energy Environ. Sci. 2014, 7, 513. doi: 10.1039/C3EE40795K

(6) Lin, D.; Liu, Y.; Cui, Y. Nat. Nanotechnol. 2017, 12, 194. doi: 10.1038/nnano.2017.16

(7) Chen, L.; Fan, X.; Ji, X.; Chen, J.; Hou, S.; Wang, C. Joule 2019, 3,
732. doi: $10.1016 /$ j.joule.2018.11.025

(8) Yang, C.; Yin, Y.; Zhang, S.; Li, N.; Guo, Y. Nat. Commun. 2015, 6, 1. doi: $10.1038 /$ ncomms 9058

(9) Duan, H.; Zhang, J.; Chen, X.; Zhang, X.; Li, J.; Huang, L.; Zhang, X.; Shi, J.; Yin, Y.; Zhang, Q. J. Am. Chem. Soc. 2018, 140, 18051. doi: $10.1021 /$ jacs.8b10488

(10) Cheng, X.; Zhang, R.; Zhao, C.; Zhang, Q. Chem. Rev. 2017, 117, 10403. doi: 10.1021/acs.chemrev.7b00115

(11) Wang, D.; Zhang, W.; Zheng, W.; Cui, X.; Rojo, T.; Zhang, Q. Adv. Sci. 2017, 4, 1600168. doi: 10.1002/advs.201600168

(12) Bouchet, R. Nat. Nanotechnol. 2014, 9, 572. doi: $10.1038 /$ nnano.2014.165

(13) Jo, H.; Song, D.; Jeong, Y.; Lee, Y. M.; Ryou, M. J. Power Sources 2019, 409, 132. doi: 10.1016/j.jpowsour.2018.09.059

(14) Xu, R.; Zhang, X. Q.; Cheng, X. B.; Peng, H. J.; Zhao, C. Z.; Yan, C.; Huang, J. Q. Adv. Funct. Mater. 2018, 28, 1705838. doi: 10.1002/adfm.201705838

(15) Zhu, J.; Li, P.; Chen, X.; Legut, D.; Fan, Y.; Zhang, R.; Lu, Y.; Cheng, X.; Zhang, Q. Energy Storage Mater. 2019, 16, 426. doi: 10.1016/j.ensm.2018.06.023

(16) Zheng, J.; Li, H. Energy Storage Sci. Tech. 2013, 5, 503. [郑杰允, 李 泓. 储能科学与技术, 2013, 5,503 .] doi: 10.3969/j.issn.2095-4239.2013.05.009

(17) Goodenough, J. B.; Kim, Y. Chem. Mater. 2009, $22,587$. doi: $10.1021 / \mathrm{cm} 901452 \mathrm{z}$

(18) Joho, F.; Rykart, B.; Blome, A.; Novák, P.; Wilhelm, H.; Spahr, M. E. J. Power Sources 2001, 97, 78. doi: 10.1016/S0378-7753(01)00595-X

(19) Peled, E. J. Electrochem. Soc. 1979, 126, 2047. doi: $10.1149 / 1.2128859$

(20) Schlaikjer, C. R.; Liang, C. C. J. Electrochem. Soc. 1971, 118, 1447 doi: $10.1149 / 1.2408351$

(21) Peled, E.; Golodnitsky, D.; Ardel, G. J. Electrochem. Soc. 1997, 144, L208. doi: 10.1149/1.1837858

(22) Zhang, Q.; Pan, J.; Lu, P.; Liu, Z.; Verbrugge, M. W.; Sheldon, B W.; Cheng, Y.; Qi, Y.; Xiao, X. Nano Lett. 2016, 16, 2011. doi: 10.1021/acs.nanolett.5b05283

(23) Aurbach, D.; Markovsky, B.; Levi, M. D.; Levi, E.; Schechter, A.; Moshkovich, M.; Cohen, Y. J. Power Sources 1999, 81, 95. doi: 10.1016/S0378-7753(99)00187-1

(24) Ein-Eli, Y. Electrochem. Solid-State Lett. 1999, $2,212$. doi: $10.1149 / 1.1390787$

(25) Whittingham, M. S. Science 1976, 192, 1126. doi: $10.1126 /$ science.192.4244.1126

(26) Nishikawa, K.; Mori, T.; Nishida, T.; Fukunaka, Y.; Rosso, M.; Homma, T. J. Electrochem. Soc. 2010, 157, A1212. 
doi: $10.1149 / 1.3486468$

(27) Ling, C.; Banerjee, D.; Matsui, M. Electrochim. Acta 2012, 76, 270. doi: 10.1016/j.electacta.2012.05.001

(28) Jäckle, M.; Groß, A. J. Chem. Phys. 2014, 141, 174710. doi: $10.1063 / 1.4901055$

(29) Ely, D. R.; García, R. E. J. Electrochem. Soc. 2013, 160, A662. doi: $10.1149 / 1.057304$ jes

(30) Yan, K.; Lu, Z.; Lee, H.; Xiong, F.; Hsu, P.; Li, Y.; Zhao, J.; Chu, S.; Cui, Y. Nat. Energy 2016, 1, 16010. doi: 10.1038/nenergy.2016.10

(31) Liu, M.; Kutana, A.; Liu, Y.; Yakobson, B. I. J. Phys. Chem. Lett. 2014, 5, 1225. doi: 10.1021/jz500199d

(32) Pei, A.; Zheng, G.; Shi, F.; Li, Y.; Cui, Y. Nano Lett. 2017, 17, 1132. doi: 10.1021/acs.nanolett.6b04755

(33) Fleury, V.; Chazalviel, J.; Rosso, M.; Sapoval, B. J. Electroanal. Chem. 1990, 290, 249. doi: 10.1016/0022-0728(90)87434-1

(34) Chazalviel, J. Phys. Rev. A 1990, 42, 7355. doi: 10.1103/PhysRevA.42.7355

(35) Rosso, M.; Brissot, C.; Teyssot, A.; Dollé, M.; Sannier, L.; Tarascon, J.; Bouchet, R.; Lascaud, S. Electrochim. Acta 2006, 51, 5334. doi: 10.1016/j.electacta.2006.02.004

(36) Bai, P.; Li, J.; Brushett, F. R.; Bazant, M. Z. Energy Environ. Sci. 2016, 9, 3221. doi: 10.1039/c6ee 01674j

(37) Sacci, R. L.; Dudney, N. J.; More, K. L.; Parent, L. R.; Arslan, I.; Browning, N. D.; Unocic, R. R. Chem. Commun. 2014, 50, 2104. doi: $10.1039 / \mathrm{C} 3 \mathrm{CC} 49029 \mathrm{G}$

(38) Kushima, A.; So, K. P.; Su, C.; Bai, P.; Kuriyama, N.; Maebashi, T.; Fujiwara, Y.; Bazant, M. Z.; Li, J. Nano Energy 2017, 32, 271. doi: 10.1016/j.nanoen.2016.12.001

(39) Bieker, G.; Winter, M.; Bieker, P. Phys. Chem. Chem. Phys. 2015, 17, 8670. doi: 10.1039/C4CP05865H

(40) Wu, B.; Lochala, J.; Taverne, T.; Xiao, J. Nano Energy 2017, 40, 34 doi: 10.1016/j.nanoen.2017.08.005

(41) Liu, G.; Lu, W. J. Electrochem. Soc. 2017, 164, A1826. doi: $10.1149 / 2.0381709$ jes

(42) Chen, L.; Li, X. L.; Zhao, Q.; Cai, W. B.; Jiang, Z. Y. Acta Phys. Chim. Sin. 2006, 22, 1155. [陈玲, 李雪莉, 赵强, 蔡文斌, 江志裕. 物理化学学报, 2006, 22, 1155.] doi: 10.3866/PKU.WHXB20060924

(43) Hong, Z.; Viswanathan, V. ACS Energy Lett. 2019, 4, 1012. doi: 10.1021/acsenergylett.9b00433

(44) Zou, P.; Wang, Y.; Chiang, S.; Wang, X.; Kang, F.; Yang, C. Nat. Commun. 2018, 9, 1. doi: 10.1038/s41467-018-02888-8

(45) Wang, C.; Appleby, A. J.; Little, F. E. J. Electroanal. Chem. 2002, 519, 9. doi: 10.1016/S0022-0728(01)00708-2

(46) Wu, H.; Cui, Y. Nano Today 2012, 7, 414. doi: 10.1016/j.nantod.2012.08.004

(47) Li, Z.; Huang, J.; Liaw, B. Y.; Metzler, V.; Zhang, J. J. Power
Sources 2014, 254, 168. doi: 10.1016/j.jpowsour.2013.12.099

(48) Chen, K.; Wood, K. N.; Kazyak, E.; LePage, W. S.; Davis, A. L.; Sanchez, A. J.; Dasgupta, N. P. J. Mater. Chem. A 2017, 5, 11671. doi: 10.1039/C7TA00371D

(49) Gireaud, L.; Grugeon, S.; Laruelle, S.; Yrieix, B.; Tarascon, J. Electrochem. Commun. 2006, 8, 1639. doi: 10.1016/j.elecom.2006.07.037

(50) Yue, X.; Li, X.; Wang, W.; Chen, D.; Qiu, Q.; Wang, Q.; Wu, X.; Fu, Z.; Shadike, Z.; Yang, X. Nano Energy 2019, 60, 257. doi: 10.1016/j.nanoen.2019.03.057

(51) Yue, X.; Bao, J.; Yang, S.; Luo, R.; Wang, Q.; Wu, X.; Shadike, Z.; Yang, X.; Zhou, Y. Nano Energy 2020, 71, 104614. doi: 10.1016/j.nanoen.2020.104614

(52) Li, L.; Basu, S.; Wang, Y.; Chen, Z.; Hundekar, P.; Wang, B.; Shi, J.; Shi, Y.; Narayanan, S.; Koratkar, N. Science 2018, 359, 1513. doi: $10.1126 /$ science.aap 8787

(53) Wu, C.; Huang, H.; Lu, W.; Wei, Z.; Ni, X.; Sun, F.; Qing, P.; Liu, Z.; Ma, J.; Wei, W. Adv. Sci. 2020, 7, 1902643. doi: 10.1002/advs.201902643

(54) Zhang, X. Q.; Chen, X.; Cheng, X. B.; Li, B. Q.; Shen, X.; Yan, C.; Huang, J. Q.; Zhang, Q. Angew. Chem. Int. Ed. 2018, 57, 5301. doi: 10.1002/ange.201803003

(55) Rong, G.; Zhang, X.; Zhao, W.; Qiu, Y.; Liu, M.; Ye, F.; Xu, Y.; Chen, J.; Hou, Y.; Li, W. Adv. Mater. 2017, 29, 1606187. doi: 10.1002/adma.201606187

(56) Golozar, M.; Hovington, P.; Paolella, A.; Bessette, S.; Lagacé, M.; Bouchard, P.; Demers, H.; Gauvin, R.; Zaghib, K. Nano Lett. 2018, 18, 7583. doi: 10.1021/acs.nanolett.8b03148

(57) Kim, S. H.; Kim, K.; Choi, H.; Im, D.; Heo, S.; Choi, H. S. J. Mater. Chem. A 2019, 7, 13650. doi: 10.1039/C9TA02614B

(58) Hou, C.; Han, J.; Liu, P.; Yang, C.; Huang, G.; Fujita, T.; Hirata, A.; Chen, M. Adv. Energy Mater. 2019, 9, 1902675. doi: 10.1002/aenm.201902675

(59) Liu, B.; Xu, W.; Tao, J.; Yan, P.; Zheng, J.; Engelhard, M. H.; Lu, D.; Wang, C.; Zhang, J. G. Adv. Energy Mater. 2018, 8, 1702340. doi: 10.1002/aenm.201702340

(60) Shen, C.; Hu, G.; Cheong, L. Z.; Huang, S.; Zhang, J. G.; Wang, D. Small Methods 2018, 2, 1700298. doi: 10.1002/smtd.201700298

(61) Kitta, M.; Sano, H. Langmuir 2017, 33, 1861. doi: 10.1021/acs.langmuir.6b04651

(62) Zhang, L.; Yang, T.; Du, C.; Liu, Q.; Tang, Y.; Zhao, J.; Wang, B.; Chen, T.; Sun, Y.; Jia, P. Nat. Nanotechnol. 2020, 15, 94. doi: 10.1038/s41565-019-0604-x

(63) Sun, F.; Zhou, D.; He, X.; Osenberg, M.; Dong, K.; Chen, L.; Mei, S.; Hilger, A.; Markötter, H.; Lu, Y. ACS Energy Lett. 2020, 5, 152. doi: 10.1021/acsenergylett.9b02424 
(64) Wenzel, S.; Leichtweiss, T.; Krüger, D.; Sann, J.; Janek, J. Solid State Ion. 2015, 278, 98. doi: 10.1016/j.ssi.2015.06.001

(65) Shen, X.; Li, Y.; Qian, T.; Liu, J.; Zhou, J.; Yan, C.; Goodenough, J. B. Nat. Commun. 2019, 10, 1. doi: 10.1038/s41467-019-08767-0

(66) Li, H.; Chao, D.; Chen, B.; Chen, X.; Chuah, C.; Tang, Y.; Jiao, Y.; Jaroniec, M.; Qiao, S. J. Am. Chem. Soc. 2020, 142, 2012. doi: $10.1021 /$ jacs.9b11774

(67) Zhang, X.; Zhang, C.; Liu, Z.; Liu, L.; Xia, L. Sci. Tech. Eng. 2019, 19, 9. [张晓凯, 张丛丛, 刘忠民, 刘力嘉, 夏蕾. 科学技术与工程, 2019, 19, 9.]

(68) Wang, X.; Li, Y.; Meng, Y. S. Joule 2018, 2, 2225. doi: 10.1016/j.joule.2018.10.005

(69) Li, Y.; Li, Y.; Pei, A.; Yan, K.; Sun, Y.; Wu, C.; Joubert, L.; Chin, R.; Koh, A. L.; Yu, Y. Science 2017, 358, 506. doi: $10.1126 /$ science.aam6014

(70) Xu, Y.; Wu, H.; He, Y.; Chen, Q.; Zhang, J. G.; Xu, W.; Wang, C. Nano Lett. 2020, 20, 418. doi: 10.1021/acs.nanolett.9b04111

(71) Zachman, M. J.; Tu, Z.; Choudhury, S.; Archer, L. A.; Kourkoutis, L. F. Nature 2018, 560, 345. doi: 10.1038/s41586-018-0397-3

(72) Wang, J.; Huang, W.; Pei, A.; Li, Y.; Shi, F.; Yu, X.; Cui, Y. Nat. Energy 2019, 4, 664. doi: 10.1038/s41560-019-0413-3
(73) Downing, R. G.; Lamaze, G. P.; Langland, J. K.; Hwang, S. T. J. Res. Natl. Inst. Stand Technol. 1993, 98, 109. doi: 10.6028/jres.098.008

(74) Han, F.; Westover, A. S.; Yue, J.; Fan, X.; Wang, F.; Chi, M.; Leonard, D. N.; Dudney, N. J.; Wang, H.; Wang, C. Nat. Energy 2019, 4, 187. doi: 10.1038/s41560-018-0312-z

(75) Lv, S.; Verhallen, T.; Vasileiadis, A.; Ooms, F.; Xu, Y.; Li, Z.; Li, Z.; Wagemaker, M. Nat. Commun. 2018, 9, 1. doi: 10.1038/s41467-018-04394-3

(76) Wang, C.; Gong, Y.; Dai, J.; Zhang, L.; Xie, H.; Pastel, G.; Liu, B.; Wachsman, E.; Wang, H.; Hu, L. J. Am. Chem. Soc. 2017, 139, 14257. doi: $10.1021 /$ jacs. $7 \mathrm{~b} 07904$

(77) Li, Q.; Yi, T.; Wang, X.; Pan, H.; Quan, B.; Liang, T.; Guo, X.; Yu, X.; Wang, H.; Huang, X. Nano Energy 2019, 63, 103895. doi: 10.1016/j.nanoen.2019.103895

(78) Zheng, J.; Tang, M.; Hu, Y. Y. Angew. Chem. Int. Ed. 2016, 55, 12538. doi: 10.1002/anie.201607539

(79) Leskes, M.; Drewett, N. E.; Hardwick, L. J.; Bruce, P. G.; Goward, G. R.; Grey, C. P. Angew. Chem. Int. Ed. 2012, 51, 8560. doi: 10.1002/anie. 201202183

(80) Leskes, M.; Moore, A. J.; Goward, G. R.; Grey, C. P. J. Phys. Chem. C 2013, 117, 26929. doi: 10.1021/jp410429k 